\title{
Jet transport-based nonlinear state and parameter estimation for geostationary spacecraft
}

\author{
Jianlin Chen ${ }^{\mathrm{a}, \mathrm{b}, \mathrm{c}, *}$, Josep J. Masdemont ${ }^{\mathrm{c}}$, Gerard Gómez ${ }^{\mathrm{d}}$, Jianping Yuanª,b,* \\ ${ }^{a}$ National Key Laboratory of Aerospace Flight Dynamics, Xi'an, Shaanxi, 710072, China \\ ${ }^{b}$ School of Astronautics, Northwestern Polytechnical University, Xi'an, Shaanxi, 710072, \\ China \\ ${ }^{c}$ IEEC \& Departament de Matemàtiques, Universitat Politècnica de Catalunya, Diagonal \\ 647, 08028 Barcelona \\ ${ }^{d}$ IEEC \& Departament de Matemàtiques i Informàtica, Universitat de Barcelona, Gran \\ Via de les Corts Catalanes 585, 08007 Barcelona
}

\begin{abstract}
Based on the jet transport technique, this paper proposes a novel nonlinear Kalman filter for simultaneously estimating the spacecraft state vector and uncertain parameters, either physically related with the spacecraft or with the measurement procedure. Two different coordinate representations, including Cartesian and hybrid geostationary orbital elements, are exploited in the new nonlinear estimators. The performance and sensitivity analyses of the proposed jet transport-based nonlinear estimators are assessed by numerical simulations and compared with the classical extended Kalman filter.
\end{abstract}

Keywords: jet transport, parameter estimation, nonlinear estimator, geostationary spacecraft

\section{Introduction}

Geostationary or geosynchronous Earth Orbit (GEO) regime has the valuable characteristic that keeps the spacecraft at rest relative to the Earthcentered Earth-fixed (ECEF) frame. This particular feature reduces the difficulty for providing long-time continuous direct broadcast, communication,

\footnotetext{
${ }^{*}$ Corresponding author

Email addresses: chenjl@mail.nwpu.edu.cn (Jianlin Chen), jyuan@nwpu.edu.cn (Jianping Yuan)
} 
and observation services for a certain fixed region of the Earth's surface. A precise initial GEO orbit determination (IOD) is of extreme importance for these space-related missions and, in particular, emerging high-accuracy GEO applications are being proposed to monitor the land stability subjected to natural hazards, like volcanic activities and earthquakes (future GEOSAR missions [1]), as well as to implement meso-scale measurements of sea altimetry using TV signals from GEO satellites which, being much stronger than the GPS ones, provide several advantages [2].

Currently, various filtering algorithms have been proposed and extensively exploited in the IOD problems [3-7]. A complete discussion of the pros and cons of these filtering algorithms can be found in $[3,8]$. The well-known extended Kalman filter (EKF) behaves well in the linear or weak nonlinear estimation problem [9]. However, it loses accuracy, or even diverges, in some certain cases such as: when the problem of state estimation is highly nonlinear, in systems with low frequency measurements, or when the initial state estimation errors are large. The unscented Kalman filter (UKF) overcomes this drawback by removing the linearization assumption but with a higher computational cost [6].

A better nonlinear extended Kalman filtering algorithm was proposed by Park and Scheeres [7] by means of a semi-analytic uncertainty prediction technique to describe the localized nonlinear motion around the nominal trajectory, and carrying out a high order Taylor expansion of the nonlinear measurements. The bottleneck of these high order Kalman filters (HEKF) is the computational complexity of the required high order derivatives that limits its applications.

Making use of the fact that Differential Algebra (DA) techniques determine high order derivatives in an accurate and efficient way, Valli et al. [3] developed a high order EKF algorithm with the use of DA for orbit determination in two simple cases: Sun-Earth halo orbit determination, and Keplerian orbit determination around the Earth. Further usages of this high order EKF in the angles-only initial orbit determination problem [4] and spacecraft relative state estimation problem [5] also validated its effectiveness. However, most of the references only consider the state estimation and leave aside the estimation of parameter uncertainties in inaccurate dynamical models or in measurement models, such as the spacecraft area-to-mass ratio, or the uncertain location of a removable ground tracking station. A simultaneous estimation of these additional parameters is meaningful to further improve the estimation accuracy of trajectories; for instance reference [10] 
underlined that the solar radiation pressure (SRP) modeling error is the largest dynamical error source in the process of the geosynchronous navigation satellites orbit determination problem, a precise area-to-mass ratio estimation can provide good a priori information for the subsequent accurate trajectory determination.

The purpose of this paper is to implement a precise geostationary or geosynchronous orbit determination, taking into account the dominant perturbations in the GEO regime. At the same time, the procedure enables to estimate additional uncertain parameters in the equations of motion or in the measurement equations, which allows to further improve the accuracy of the models and of the orbit determination solution. The methodology is systematically implemented and can be easily extended for any parameter estimation or orbit determination problem in another regime.

The paper is organized as follows: Section 2 describes the equations of motion used in this work, including a Cartesian representation and a hybrid GEO element representation. In Section 3 we develop the jet transport-based HEKF algorithms for a spacecraft physical parameters estimation, and for a ground tracking station position estimation, using both kinds of coordinates. Section 4 contains some of the simulations done to validate the efficiency of the estimators. The conclusions are given in Section 5 .

\section{Dynamical model}

Several coordinate representations were proposed in the past to describe the motion of GEO satellites [11]. However, the particularity of GEO orbits leads specially to two main representations for the description of its motion without singularities. They are the usual Cartesian representation and the particular GEO element representation [12], that will be both considered in this work.

\subsection{Cartesian representation}

Consider the motion of a spacecraft close to the GEO regime subjected to the central gravitational acceleration of an homogeneous spherical Earth $\boldsymbol{a}_{c e n}=\left(\frac{-\mu}{r^{3}} x, \frac{-\mu}{r^{3}} y, \frac{-\mu}{r^{3}} z\right)^{T}$ and to the four main perturbing accelerations: $\boldsymbol{a}_{n o n}, \boldsymbol{a}_{S R P}, \boldsymbol{a}_{s}$, and $\boldsymbol{a}_{m}$, that respectively denote perturbing Earth's nonspherical gravitational acceleration, solar radiation pressure, Sun and Moon 
gravitational accelerations [9]. The equations of motion in Cartesian coordinates are

$$
\begin{aligned}
\ddot{\boldsymbol{r}} & =\boldsymbol{a}_{c e n}+\boldsymbol{a}_{p}, \\
\boldsymbol{a}_{p} & =\boldsymbol{a}_{n o n}+\boldsymbol{a}_{S R P}+\boldsymbol{a}_{s}+\boldsymbol{a}_{m},
\end{aligned}
$$

where $\boldsymbol{r}$ indicates the spacecraft position vector in the Earth-centered inertial (ECI) frame, and $r$ is its norm, the notation $\boldsymbol{a}_{p}$ indicates the total perturbing acceleration.

The adopted formulae for the dominant perturbing accelerations can be found in detail in [9]. The SRP, Sun and Moon gravitational accelerations can be expressed in the ECI reference frame as,

$$
\begin{gathered}
\boldsymbol{a}_{S R P}^{E C I}=-\nu P C_{r} \frac{A}{m} \frac{\boldsymbol{r}_{\odot}}{r_{\odot}^{3}} A U^{2}, \\
\boldsymbol{a}_{M}^{E C I}=G M\left(\frac{\boldsymbol{r}_{M}-\boldsymbol{r}}{\left|\boldsymbol{r}_{M}-\boldsymbol{r}\right|^{3}}-\frac{\boldsymbol{r}_{M}}{\left|r_{M}\right|^{3}}\right),
\end{gathered}
$$

where $\boldsymbol{r}_{\odot}$ is the relative position vector pointing from the Sun to the spacecraft, $P$ indicates the SRP force at a distance of 1 AU, $\nu$ denotes the Earth's shadow function and $M$ represents the mass of the Sun or Moon, $\boldsymbol{r}_{M}$ is the position vector of the mass $M$ relative to the Earth. Additional parameters, including the radiation pressure coefficient $C_{r}$, the spacecraft mass $m$, and the cross sectional area $A$, are related to the individual spacecraft properties. Note that all these spacecraft physical parameters can be affected by some uncertainties, and can be estimated by the nonlinear filters introduced in this work.

The adopted Earth's non-spherical gravity is expressed in the ECEF frame as,

$$
\boldsymbol{a}_{n o n}^{E C E F}=\left(\sum_{n, m} a_{x, n m}+\frac{\mu}{r^{3}} x, \sum_{n, m} a_{y, n m}+\frac{\mu}{r^{3}} y, \sum_{n, m} a_{z, n m}+\frac{\mu}{r^{3}} z,\right),
$$


where

$$
\begin{aligned}
a_{x, n m}= & \frac{\mu}{2 R_{\oplus}^{2}}\left\{\left(-C_{n m} V_{n+1, m+1}-S_{n m} W_{n+1, m+1}\right)+\right. \\
& \left.\frac{(n-m+2) !}{(n-m) !}\left(C_{n m} V_{n+1, m-1}+S_{n m} W_{n+1, m-1}\right)\right\}, \\
a_{y, n m}= & \frac{\mu}{2 R_{\oplus}^{2}}\left\{\left(-C_{n m} W_{n+1, m+1}+S_{n m} V_{n+1, m+1}\right)+\right. \\
& \left.\frac{(n-m+2) !}{(n-m) !}\left(-C_{n m} W_{n+1, m-1}+S_{n m} V_{n+1, m-1}\right)\right\}, \\
a_{z, n m}= & \frac{\mu}{R_{\oplus}^{2}}\left\{(n-m+1)\left(-C_{n m} V_{n+1, m}-S_{n m} W_{n+1, m}\right)\right\} .
\end{aligned}
$$

In these equations the Earth's equatorial radius $R_{\oplus}$, and the geopotential coefficients $C_{n m}, S_{n m}$ are provided by the $5 \times 5$ EGM96S gravity model. In particular, $m=n=0$ corresponds to the situation in which only the central gravitational acceleration is considered. The values of $V_{n m}$ and $W_{n m}$ are recursively given by

$$
\begin{aligned}
V_{00} & =\frac{R_{\oplus}}{r}, \quad V_{m-1, m}=0, \\
V_{m m} & =(2 m-1) \frac{R_{\oplus}}{r^{2}}\left\{x V_{m-1, m-1}-y W_{m-1, m-1}\right\} \\
V_{n m} & =\left(\frac{2 n-1}{n-m}\right) \frac{z R_{\oplus}}{r^{2}} V_{n-1, m}-\left(\frac{n+m-1}{n-m}\right) \frac{R_{\oplus}^{2}}{r^{2}} V_{n-2, m}, \\
W_{00} & =0, \quad W_{m-1, m}=0, \\
W_{m m} & =(2 m-1) \frac{R_{\oplus}}{r^{2}}\left\{x W_{m-1, m-1}+y V_{m-1, m-1}\right\} \\
W_{n m} & =\left(\frac{2 n-1}{n-m}\right) \frac{z R_{\oplus}}{r^{2}} W_{n-1, m}-\left(\frac{n+m-1}{n-m}\right) \frac{R_{\oplus}^{2}}{r^{2}} W_{n-2, m} .
\end{aligned}
$$

Besides, the time-dependent coordinate transformation from the ECEF frame to the ECI frame can be obtained via the use of average angular speed of the Earth's rotation $\omega_{e}=7.292115 \times 10^{-5} \mathrm{rad} / \mathrm{s}$, thus

$$
\mathcal{T}_{E C E F}^{E C I}=\left(\begin{array}{ccc}
\cos \left(\omega_{e} t\right) & -\sin \left(\omega_{e} t\right) & 0 \\
\sin \left(\omega_{e} t\right) & \cos \left(\omega_{e} t\right) & 0 \\
0 & 0 & 1
\end{array}\right)
$$


Therefore, the perturbing Earth's non-spherical gravitational acceleration is expressed in the ECI reference frame as

$$
\boldsymbol{a}_{\text {non }}^{E C I}=\mathcal{T}_{E C E F}^{E C I} \cdot \boldsymbol{a}_{\text {non }}^{E C E F} .
$$

\subsection{GEO representation}

In [13], Tombasco introduced a non-dimensional GEO element set in terms of the classical Keplerian elements $\{a, e, i, \omega, \Omega, \theta\}$, which consists of the Earth-fixed sub-spacecraft longitude $\lambda$, the longitudinal drift rate $\delta \bar{a}$, two eccentricity vector components $\left(e_{x}, e_{y}\right)$, and two equinoctial elements $\left(Q_{1}, Q_{2}\right)$. The expressions of the GEO elements, as well as four intermediate variables, can be written as follows,

$$
\begin{aligned}
\lambda & \triangleq(\omega+\Omega+\theta)-G A(t), \\
\delta \bar{a} & \triangleq \frac{a-A_{n}}{A_{n}}, \\
e_{x} & \triangleq e \cos (\omega+\Omega), \\
e_{y} & \triangleq e \sin (\omega+\Omega), \\
Q_{1} & \triangleq \tan \left(\frac{i}{2}\right) \sin (\Omega), \\
Q_{2} & \triangleq \tan \left(\frac{i}{2}\right) \cos (\Omega), \\
r & =\frac{A_{n}(\delta \bar{a}+1)\left(1-e_{x}^{2}-e_{y}^{2}\right)}{1+e_{x} \cos s+e_{y} \sin s}, \\
s & =\lambda+G A(t)=\omega+\Omega+\theta, \\
p & =A_{n}(\delta \bar{a}+1)\left(1-e_{x}^{2}-e_{y}^{2}\right), \\
h & =\sqrt{p \mu},
\end{aligned}
$$

where $A_{n}=42164.2 \mathrm{~km}$ indicates a nominal GEO semi-major axis, $G A(t)=$ $G A\left(t_{0}\right)+\omega_{e}\left(t-t_{0}\right)$ stands for the Greenwich sidereal angle at $t$. The notation $s$ denotes the spacecraft sidereal angle, $p$ represents the semi-latus rectum of the orbit, and $h$ is the norm of the angular momentum. With the above 
variables, the equations of motion using GEO element representation are

$$
\begin{aligned}
\dot{\lambda}= & \frac{h}{r^{2}}+\frac{r}{h}\left[Q_{2} \sin s-Q_{1} \cos s\right] a_{h}-\omega_{e}, \\
\delta \dot{\bar{a}}= & \frac{2(\delta \bar{a}+1)^{2}}{h A_{n}}\left[\left(e_{x} \sin s-e_{y} \cos s\right) a_{r}+\frac{p}{r} a_{\theta}\right], \\
\dot{e}_{x}= & \frac{r}{h}\left\{\frac{p}{r} \sin s \cdot a_{r}+\left[e_{x}+\left(1+\frac{p}{r}\right) \cos s\right] a_{\theta}+e_{y}\left[Q_{1} \cos s\right.\right. \\
& \left.\left.-Q_{2} \sin s\right] a_{h}\right\}, \\
\dot{e}_{y}= & \frac{r}{h}\left\{-\frac{p}{r} \cos s \cdot a_{r}+\left[e_{y}+\left(1+\frac{p}{r}\right) \sin s\right] a_{\theta}-e_{x}\left[Q_{1} \cos s\right.\right. \\
& \left.\left.-Q_{2} \sin s\right] a_{h}\right\}, \\
\dot{Q}_{1}= & \frac{r}{2 h}\left(1+Q_{1}^{2}+Q_{2}^{2}\right) \sin s \cdot a_{h}, \\
\dot{Q}_{2}= & \frac{r}{2 h}\left(1+Q_{1}^{2}+Q_{2}^{2}\right) \cos s \cdot a_{h} .
\end{aligned}
$$

We note that, using Cartesian coordinates, the perturbing accelerations in (1) can be easily computed through (2), (3), and (4). However, when we implement the nonlinear estimation using GEO elements, a series of conversions have to be done for computing $\boldsymbol{a}_{p}=\left(a_{r}, a_{\theta}, a_{h}\right)^{T}$ in (5). It is as follows: i) at a certain epoch transform the known GEO elements into Cartesian coordinates (the transformation is given in [14]); ii) compute the dominant perturbing accelerations using the Cartesian coordinates through (2), (3), and (4); iii) project all the perturbations into the Local Vertical Local Horizontal reference frame (LVLH). For the transformation of accelerations from the ECI into to the LVLH frame we make use of the cosines matrix

$$
\mathcal{T}_{E C I}^{L V L}=\left(\begin{array}{ccc}
\boldsymbol{I} \cdot \boldsymbol{i} & \boldsymbol{I} \cdot \boldsymbol{j} & \boldsymbol{I} \cdot \boldsymbol{k} \\
\boldsymbol{J} \cdot \boldsymbol{i} & \boldsymbol{J} \cdot \boldsymbol{j} & \boldsymbol{J} \cdot \boldsymbol{k} \\
\boldsymbol{K} \cdot \boldsymbol{i} & \boldsymbol{K} \cdot \boldsymbol{j} & \boldsymbol{K} \cdot \boldsymbol{k}
\end{array}\right)
$$

where $\boldsymbol{I}, \boldsymbol{J}, \boldsymbol{K}$ and $\boldsymbol{i}, \boldsymbol{j}, \boldsymbol{k}$ are the unitary vector sets defining the ECI and LVLH reference frames respectively. Using the spacecraft state $(\boldsymbol{r}, \boldsymbol{v})$ in the ECI frame, we have,

$$
\boldsymbol{i}=\frac{\boldsymbol{r}}{\|\boldsymbol{r}\|}, \quad \boldsymbol{k}=\frac{\boldsymbol{r} \times \boldsymbol{v}}{\|\boldsymbol{r} \times \boldsymbol{v}\|}, \quad \boldsymbol{j}=\boldsymbol{k} \times \boldsymbol{i} .
$$

Thus, the four dominant perturbations we consider can be rotated into the LVLH frame by means of

$$
\boldsymbol{a}_{p}^{L V L H}=\mathcal{T}_{E C I}^{L V L H} \cdot \boldsymbol{a}_{p}^{E C I} .
$$


Finally, a jet transport-based numerical integrator is developed using $\kappa$ th order Taylor expansions (JT-HEKF- $\kappa$ algorithm) in subsection 3.2. It is used to propagate both dynamic models and produce the spacecraft state and parameter predictions at a new epoch; then, the JT-HEKF- $\kappa$ filtering algorithms do the state and parameter updates by incorporating the new measurements. The proposed JT-HEKF- $\kappa$ filtering algorithms make an intensive use of this procedure to estimate the state vector and parameters in the GEO element space.

\section{Jet transport technique}

The DA technique, as a way for doing efficient symbolic computations, was first proposed by Berz and Makino [15] in 1999 for the study of particle beam accelerators, and implemented by the same authors in the COSY Infinity package. With similar ideas, Pérez-Palau [16] studied the application of JT technique in the structure detection of astrodynamics problem. Essentially, either DA method or JT method is an automatic differentiation technique that enables to derive high order Taylor expansions (or other kind of expansions) of general nonlinear functions and, in particular, of the flow associated to an ODE in an accurate and efficient way. For convenience of the reader, some vital informations on jet transport technique are reviewed in this section. More fundamental details about Jet Transport formulation, as well as its computer implementation can be found in $[16,17]$.

\subsection{Jet transport nonlinear expansion}

Consider an ODE system $\dot{\boldsymbol{x}}=\boldsymbol{f}(\boldsymbol{x}, \boldsymbol{p}, t)$, such as the ones given by (1) or (5), with a $n$-dimensional state vector $\boldsymbol{x}$, and a $l$-dimensional parameter vector $\boldsymbol{p}$, as well as the initial conditions $\boldsymbol{x}\left(t_{0}\right)=\boldsymbol{x}_{0}$, and $\boldsymbol{p}\left(t_{0}\right)=\boldsymbol{p}_{0}$. The JT method performs the propagation of a neighborhood $\mathcal{N}_{0}$ around $\boldsymbol{x}_{0}$ and $\boldsymbol{p}_{0}$ from $t_{0}$ up to a final time $t_{d}$.

A sketch of the procedure is the following: first parameterize the initial neighborhood $\mathcal{N}_{0}$ by a pair of polynomial vectors $\left[\boldsymbol{x}_{0}\right]=\overline{\boldsymbol{x}}_{0}+\delta \boldsymbol{x}_{0}$ and $\left[\boldsymbol{p}_{0}\right]=\overline{\boldsymbol{p}}_{0}+\delta \boldsymbol{p}_{0}$, where $\overline{\boldsymbol{x}}_{0}$ and $\overline{\boldsymbol{p}}_{0}$ are the initial nominal values, while $\delta \boldsymbol{x}_{0}$ and $\delta \boldsymbol{p}_{0}$ are their initial uncertainties. Then, the initial state and parameter uncertainty neighborhood $\mathcal{N}_{0}$ is integrated in the accurate nonlinear vectorfield, that includes the Earth potential and perturbations, from the initial epoch $t_{0}$ up to a final epoch $t_{d}$, using a JT-based eighth order variable step Runge 
Kutta method, or by means of any other numerical integrator. The result is a $\kappa$-th order state polynomial vector, $\left[\boldsymbol{x}_{1}\right]^{\kappa}=\overline{\boldsymbol{x}}_{d}+\mathcal{P}_{\boldsymbol{x}_{d}}^{\kappa}\left(\delta \boldsymbol{x}_{0}, \delta \boldsymbol{p}_{0}\right)$, which approximates the state uncertainty neighborhood $\boldsymbol{\Phi}\left(t_{d} ; t_{0}, \boldsymbol{x}_{0}+\delta \boldsymbol{x}_{0}, \boldsymbol{p}_{0}+\delta \boldsymbol{p}_{0}\right)$, where $\boldsymbol{\Phi}$ denotes the flow associated to the differential equation, and a similar representation, $\left[\boldsymbol{p}_{d}\right]=\overline{\boldsymbol{p}}_{d}+\mathcal{P}_{\boldsymbol{p}_{d}}^{\kappa}\left(\delta \boldsymbol{p}_{0}\right)$, that approximates the parameter uncertainty neighborhood at time $t_{d}$. In particular, we note that the truncated state Taylor series at time $t_{d}$ can be written as

$$
\begin{aligned}
{\left[\boldsymbol{x}_{d}\right]^{\kappa} } & =\overline{\boldsymbol{x}}_{d}+\mathcal{P}_{\boldsymbol{x}_{d}}^{\kappa}\left(\delta \boldsymbol{x}_{0}, \delta \boldsymbol{p}_{0}\right) \\
& =\sum_{0 \leq \gamma_{1}+\cdots+\gamma_{n+l} \leq \kappa} \boldsymbol{a}_{\gamma_{1} \cdots \gamma_{n+l}} \delta x_{0,1}^{\gamma_{1}} \cdots \delta x_{0, n}^{\gamma_{n}} \delta p_{0,1}^{\gamma_{n+1}} \cdots \delta p_{0, l}^{\gamma_{n+l}}
\end{aligned}
$$

where $\delta \boldsymbol{x}_{0}=\left(\delta x_{0,1}, \ldots, \delta x_{0, n}\right)^{T}, \delta \boldsymbol{p}_{0}=\left(\delta p_{0,1}, \ldots, \delta p_{0, l}\right)^{T}$, while

$$
\boldsymbol{a}_{\gamma_{1} \ldots \gamma_{n+l}}=\frac{1}{\gamma !} \frac{\partial^{\gamma} \boldsymbol{\Phi}_{x_{d}}}{\partial \delta x_{0,1}^{\gamma_{1}} \cdots \partial \delta x_{0, n}^{\gamma_{n}} \partial \delta p_{0,1}^{\gamma_{n+1}} \cdots \partial \delta p_{0, l}^{\gamma_{n+l}}},
$$

are the coefficients of the Taylor expansion of the solution flow, and $\gamma=$ $\gamma_{1}+\cdots+\gamma_{n+l}$. Clearly, the accuracy of (8) is mainly affected by the expansion order $\kappa$, the size of $\delta \boldsymbol{x}_{0}$ and $\delta \boldsymbol{p}_{0}$, and the total propagation time $\Delta t=t_{d}-$ $t_{0}$. In principle, for a given uncertainty neighborhood, the approximation accuracy can reach a given desired tolerance by tuning the expansion orders; a detailed analysis about the procedure is given in [14]. Note that if $\kappa=1$, the JT method degrades to the linear approximation of the flow around the nominal orbit, and the usual state transition matrix is given by the coefficients $\boldsymbol{a}_{\gamma_{1} \ldots \gamma_{n+l}}$ associated to the first order of the expansion $\left(\gamma_{1}+\cdots+\right.$ $\left.\gamma_{n+l}=1\right)$.

\subsection{JT-based Runge Kutta integrator}

In order to perform a JT-based numerical integration, all the arithmetic operations of the usual numerical propagator must be replaced with the corresponding polynomial algebra in the JT scheme. After each time step, the state $\boldsymbol{x}$ and the right hand side of the ODE system $\dot{\boldsymbol{x}}=\boldsymbol{f}(\boldsymbol{x}, t)$ is expressed as a $k$ th-order expansion around a certain point of the nominal solution. At this point, it is worth to remark a particularity of the JT-based numerical integrators with adaptive step size control, such as the Runge-Kutta-Fehlberg procedures.

The Runge-Kutta methods implemented in the JT shceme (JTRK) are the only ones we consider in our study. These methods can be divided in two 
categories, including fixed or variable stepsize. In general, a JTRK integrator with variable stepsize, such as JTRK45 and JTRK78 of respective orders 5 and 8 , gives a better performance but, some care has to be taken in the stepsize control when doing JT propagations. In the following we discuss some remarks about the implementation of a JTRK78 algorithm. Other implementations, like the one of JTRK45, can be performed using similar error estimation and stepsize control strategy.

Consider the initial value problem in a vectorfield with $n$ variables. Assume a well approximated JT-state $\left[\boldsymbol{x}_{j}\right]^{k}$ at time $t_{j}$ is known. For a given time step $h$, two estimates of the real state $\boldsymbol{x}\left(t_{j+1}\right)$ at $t_{j+1}=t_{j}+h$, are provided by the seventh and eighth order JTRK, respectively:

$$
\left[\overline{\boldsymbol{x}}_{j+1}\right]^{k}=\left[\boldsymbol{x}_{j}\right]^{k}+h \boldsymbol{\phi}_{7}\left(t_{j},\left[\boldsymbol{x}_{j}\right]^{k}, h\right), \quad\left[\hat{\boldsymbol{x}}_{j+1}\right]^{k}=\left[\boldsymbol{x}_{j}\right]^{k}+h \boldsymbol{\phi}_{8}\left(t_{j},\left[\boldsymbol{x}_{j}\right]^{k}, h\right) .
$$

The fundamental idea underlying a JTRK method with variable stepsize control is that when the two estimations are close enough (this is, a suitable norm of their difference is below a selected threshold value $\epsilon$ ), the propagation with stepsize $h$ is considered successful, while when they differ, the stepsize must be recomputed and adjusted. A trade-off between a fixed accuracy and the computational time, requires also to look for the maximum stepsize which could be suitable to obtain a good estimation the real state $\boldsymbol{x}\left(t_{j+1}\right)$ when the estimators differ, as well as for the computation of $\boldsymbol{x}\left(t_{j+2}\right)$ at time $t_{j+2}$ that will follow in the propagation.

Note that, at time step $j$, the difference between the two estimations is an expansion of the form,

$$
\left[\overline{\boldsymbol{x}}_{j+1}^{i}\right]^{k}-\left[\hat{\boldsymbol{x}}_{j+1}^{i}\right]^{k}=\sum_{0 \leq m \leq k} \boldsymbol{a}_{m}^{j} \boldsymbol{\delta} \boldsymbol{x}_{m}=\sum_{0 \leq k_{1}+\cdots+k_{n} \leq k} \boldsymbol{a}_{k_{1} \ldots k_{n}}^{j} \delta x_{1}^{k_{1}} \ldots \delta x_{n}^{k_{n}} .
$$

The classical pointwise propagation algorithms estimate this difference considering some norm of the $n$-vector $\boldsymbol{a}_{0 \ldots 0}^{j}$, but in our case (10) is a $k$ thorder expansion in $n$ variables and all terms must be taken into account. Assuming a threshold for each order, $\boldsymbol{\epsilon}=\left(\epsilon_{1} \ldots \epsilon_{k}\right)$ we can define a norm by $^{1}$ :

$$
A_{m}^{j}=\max _{k_{1}+\ldots+k_{n}=m}\left\|\boldsymbol{a}_{k_{1} \ldots k_{n}}^{j}\right\|, \quad\left\|\boldsymbol{a}_{k_{1} \ldots k_{n}}^{j}\right\|=\max _{1 \leq i \leq n}\left|a_{i, k_{1} \ldots k_{n}}^{j}\right| .
$$

\footnotetext{
${ }^{1}$ Other definitions of $A_{m}^{j}$ like scaled Euclidean norms or average of absolute values among components could be also considered.
} 
The accuracy condition for a successful time step $h$ at time $t_{j}$ would be $A_{m}^{j}<\epsilon_{m}$ for $m=0 \ldots k$. In order to further determine the optimal stepsize for a given accuracy $\boldsymbol{\epsilon}$, let us first introduce:

$$
\boldsymbol{\Delta}(t, \boldsymbol{x}, h)=\frac{\boldsymbol{x}(t+h)-\boldsymbol{x}(t)}{h} .
$$

Since the JTRK7 integrator has a 7th-order local truncation error, we can write:

$$
-\boldsymbol{\Delta}\left(t_{j}, \boldsymbol{x}_{j}, h\right)+\boldsymbol{\phi}_{7}\left(t_{j},\left[\boldsymbol{x}_{j}\right]^{k}, h\right)=\boldsymbol{N}_{7}\left(t_{j}\right) h^{7}+O\left(h^{8}\right) .
$$

Analogously, the JTRK8 integrator has 8th order accuracy and thus:

$$
-\boldsymbol{\Delta}\left(t_{j}, \boldsymbol{x}_{j}, h\right)+\boldsymbol{\phi}_{8}\left(t_{j},\left[\boldsymbol{x}_{j}\right]^{k}, h\right)=\boldsymbol{N}_{8}\left(t_{j}\right) h^{8}+O\left(h^{9}\right) .
$$

Subtracting the estimations given by (9), and using (12-13), we get:

$$
\left[\overline{\boldsymbol{x}}_{j+1}\right]^{k}-\left[\hat{\boldsymbol{x}}_{j+1}\right]^{k}=h\left(\boldsymbol{\phi}_{7}-\boldsymbol{\phi}_{8}\right)=\boldsymbol{N}_{7}\left(t_{j}\right) h^{8}+O\left(h^{9}\right) .
$$

Neglecting the $O\left(h^{9}\right)$ term we obtain the following estimate of the leading coefficient in the truncation:

$$
\boldsymbol{N}_{7}\left(t_{j}\right) \simeq \frac{\left[\overline{\boldsymbol{x}}_{j+1}\right]^{k}-\left[\hat{\boldsymbol{x}}_{j+1}\right]^{k}}{h^{8}},
$$

and, according to (11), we have the following estimation for the size of the different orders of $\boldsymbol{N}_{7}\left(t_{j}\right)$ :

$$
N_{7, m}^{j}=\frac{A_{m}^{j}}{h^{8}}, \quad m=0, \ldots, k .
$$

Let us now assume a new optimal time step $h_{N}$ at time $t_{j+1}$. According to (15) together with the successful condition, we get:

$$
A_{m}^{j+1}=h_{N}^{8} N_{7, m}^{j+1} \leq \epsilon_{m}, \quad m=0, \ldots, k .
$$

Expanding $\boldsymbol{N}_{7}\left(t_{j+1}\right)$ around $t_{j}$ yields $\boldsymbol{N}_{7}\left(t_{j+1}\right)=\boldsymbol{N}_{7}\left(t_{j}+h_{N}\right)=\boldsymbol{N}_{7}\left(t_{j}\right)+$ $O\left(h_{N}\right)$, and so (16) can be written as:

$$
A_{m}^{j+1}=h_{N}^{8} N_{7, m}^{j}+O\left(h_{N}^{9}\right) \leq \epsilon_{m}, \quad m=0, \ldots, k .
$$


Then, neglecting the $O\left(h_{N}^{9}\right)$ term, and substituting (15) into (17), we obtain:

$$
h_{N, m} \leq \sqrt[8]{\frac{\epsilon_{m}}{N_{7, m}^{j}}}=h \sqrt[8]{\frac{\epsilon_{m}}{A_{m}^{j}}}, \quad m=0, \ldots, k .
$$

The value of $h_{N}$ advised for the next step propagation is the minimum of these $k+1$ values. Usually $h_{N}$ is multiplied by a safety factor of 0.9 , since, in practice, this avoids re-computations of the stepsize and increases performance.

\subsection{Jet transport-based high order extended Kalman filters}

In this subsection we propose JT-HEKF- $\kappa$ filters for the simultaneous nonlinear estimation of the spacecraft state and parameter vectors. Using either (1) or (5), the state equations of the GEO spacecraft can be discretized as

$$
\boldsymbol{x}_{k+1}=\boldsymbol{\Phi}_{x, k+1}\left(t_{k+1} ; t_{k}, \boldsymbol{x}_{k}+\delta \boldsymbol{x}_{k}, \boldsymbol{p}_{k}+\delta \boldsymbol{p}_{k}\right)+\boldsymbol{w}_{k},
$$

where $\boldsymbol{x}_{k}$ and $\boldsymbol{p}_{k}$ respectively indicate the nominal spacecraft state and parameters at time $t_{k}, \delta \boldsymbol{x}_{k}, \delta \boldsymbol{p}_{k}$ are their uncertainty deviations, and $\boldsymbol{w}_{k}$ is the process noise vector, assumed as a Gaussian white noise process, i.e., $E\left\{\boldsymbol{w}_{k}\right\}=\mathbf{0}, E\left\{\boldsymbol{w}_{k} \boldsymbol{w}_{k}^{T}\right\}=\boldsymbol{Q}_{n \times n}^{x}$. We assume the initial state estimates being a multivariable Gaussian distribution with mean $\boldsymbol{x}_{+, 0}$ and covariance $E\left\{\left(\boldsymbol{x}_{0}-\boldsymbol{x}_{+, 0}\right)\left(\boldsymbol{x}_{0}-\boldsymbol{x}_{+, 0}\right)^{T}\right\}=\boldsymbol{P}_{+, 0}^{\boldsymbol{x}}$. Moreover, it is reasonable to suppose that the spacecraft physical parameters, or the uncertain position vector of the ground tracking station, only depend on time or on the parameters themselves, in this case the discretized parameter equations are

$$
\boldsymbol{p}_{k+1}=\boldsymbol{\Phi}_{p, k+1}\left(t_{k+1} ; t_{k}, \boldsymbol{p}_{k}+\delta \boldsymbol{p}_{k}\right)+\boldsymbol{v}_{k}
$$

where $\boldsymbol{v}_{k}$ denotes the process noise vector, assumed again to be a Gaussian white noise process, i.e., $E\left\{\boldsymbol{v}_{k}\right\}=\mathbf{0}, E\left\{\boldsymbol{v}_{k} \boldsymbol{v}_{k}^{T}\right\}=\boldsymbol{Q}_{l \times l}^{p}$. It will also be assumed that the process noise vectors $\boldsymbol{w}_{k}$ and $\boldsymbol{v}_{k}$ are non-correlated, i.e. $E\left\{\boldsymbol{w}_{k}, \boldsymbol{v}_{k}\right\}=\mathbf{0}$.

Note that the parameters can be merged into the spacecraft state vector and form an augmented state vector $\boldsymbol{X}=[\boldsymbol{x}, \boldsymbol{p}]$. Thus, (18) and (19) are augmented as

$$
\boldsymbol{X}_{k+1}=\boldsymbol{\Phi}_{k+1}\left(t_{k+1} ; t_{k}, \boldsymbol{X}_{k}+\delta \boldsymbol{X}_{k}\right)+\hat{\boldsymbol{w}}_{k},
$$


where $\hat{\boldsymbol{w}}_{k}=\left[\boldsymbol{w}_{k}^{T}, \boldsymbol{v}_{k}^{T}\right]^{T}$, and the complete covariance matrix of the process noise is a $(n+l) \times(n+l)$ block diagonal matrix consisting of $\boldsymbol{Q}_{n \times n}^{\boldsymbol{x}}$ and $\boldsymbol{Q}_{l \times l}^{p}$, this is

$$
\boldsymbol{Q}=\left(\begin{array}{cc}
\boldsymbol{Q}_{n \times n}^{\boldsymbol{x}} & \mathbf{0}_{n \times l} \\
\boldsymbol{0}_{l \times n} & \boldsymbol{Q}_{l \times l}^{p}
\end{array}\right) .
$$

Assume that a measurement is obtained from the sensors in a ground tracking station at time $t_{k+1}$. The nonlinear measurement equations are written as

$$
\boldsymbol{z}_{k+1}=\boldsymbol{\Xi}_{k+1}\left(t_{k+1} ; t_{k}, \boldsymbol{X}_{k+1}\right)+\boldsymbol{u}_{k+1},
$$

where $\boldsymbol{z}_{k+1}$ is a $m$-dimensional measurement vector, and the measurement noise vector $\boldsymbol{u}_{k+1}$ is assumed to be a Gaussian white noise with mean and covariance given by $E\left\{\boldsymbol{u}_{k+1}\right\}=\mathbf{0}$, and $E\left\{\boldsymbol{u}_{k+1} \boldsymbol{u}_{k+1}^{T}\right\}=\boldsymbol{R}$. It is also assumed that the process and measurement noises are non-correlated, i.e., $E\left\{\boldsymbol{w}_{k}, \boldsymbol{u}_{k}\right\}=\mathbf{0}, E\left\{\boldsymbol{v}_{k}, \boldsymbol{u}_{k}\right\}=\mathbf{0}$.

Consider the system model equations (20) and (21), the filtering algorithms can be defined as follows:

- Prediction equations: at time $t_{k+1}$,

$$
\begin{aligned}
\boldsymbol{X}_{-, k+1}^{i}= & E\left\{\boldsymbol{\Phi}_{k+1}^{i}\left(t_{k+1} ; t_{k}, \boldsymbol{X}_{+, k}+\delta \boldsymbol{X}_{k}\right)+\hat{\boldsymbol{w}}_{k}^{i}\right\}, \\
\boldsymbol{P}_{-, k+1}^{i_{1} i_{2}}= & E\left\{\left[\boldsymbol{\Phi}_{k+1}^{i_{1}}\left(t_{k+1} ; t_{k}, \boldsymbol{X}_{+, k}+\delta \boldsymbol{X}_{k}\right)-\boldsymbol{X}_{-, k+1}^{i_{1}}+\hat{\boldsymbol{w}}_{k}^{i_{1}}\right] .\right. \\
& {\left.\left[\boldsymbol{\Phi}_{k+1}^{i_{2}}\left(t_{k+1} ; t_{k}, \boldsymbol{X}_{+, k}+\delta \boldsymbol{X}_{k}\right)-\boldsymbol{X}_{-, k+1}^{i_{2}}+\hat{\boldsymbol{w}}_{k}^{i_{2}}\right]\right\}, } \\
\boldsymbol{z}_{-, k+1}^{d}= & E\left\{\boldsymbol{\Xi}_{k+1}^{d}\left(t_{k+1} ; t_{k}, \boldsymbol{X}_{-, k+1}\right)+\boldsymbol{u}_{k+1}^{d}\right\},
\end{aligned}
$$

where $E\{\}$ represents the expectation operator; $i, i_{1}, i_{2}=1, \cdots, n$, $d=1, \cdots, m . \boldsymbol{X}_{-, k+1}^{i}$ and $\boldsymbol{P}_{-, k+1}^{i_{1} i_{2}}$ indicate the components of the mean and covariance matrix of the state; $\boldsymbol{z}_{-, k+1}^{d}$ denotes the components of the mean of the measurements.

- Update equations: we incorporate the new measurement $\boldsymbol{z}_{k+1}$ at time $t_{k+1}$ into the estimation algorithm,

$$
\begin{aligned}
\boldsymbol{P}_{z z, k+1}^{d_{1} d_{2}}= & E\left\{\left[\boldsymbol{\Xi}_{k+1}^{d_{1}}\left(t_{k+1} ; t_{k}, \boldsymbol{X}_{-, k+1}\right)-\boldsymbol{z}_{-, k+1}^{d_{1}}+\boldsymbol{u}_{k+1}^{d_{1}}\right]\right. \\
& {\left.\left[\boldsymbol{\Xi}_{k+1}^{d_{2}}\left(t_{k+1} ; t_{k}, \boldsymbol{X}_{-, k+1}\right)-\boldsymbol{z}_{-, k+1}^{d_{2}}+\boldsymbol{u}_{k+1}^{d_{2}}\right]\right\} } \\
\boldsymbol{P}_{X z, k+1}^{i d}= & E\left\{\left[\boldsymbol{\Phi}_{k+1}^{i}\left(t_{k+1} ; t_{k}, \boldsymbol{X}_{+, k}+\delta \boldsymbol{X}_{k}\right)-\boldsymbol{X}_{-, k+1}^{i}+\hat{\boldsymbol{w}}_{k}^{i}\right]\right. \\
& {\left.\left[\boldsymbol{\Xi}_{k+1}^{d}\left(t_{k+1} ; t_{k}, \boldsymbol{X}_{-, k+1}\right)-\boldsymbol{z}_{-, k+1}^{d}+\boldsymbol{u}_{k+1}^{d}\right]\right\} } \\
\boldsymbol{K}_{k+1}= & \boldsymbol{P}_{X z, k+1}\left(\boldsymbol{P}_{z z, k+1}\right)^{-1} \\
\boldsymbol{X}_{+, k+1}= & \boldsymbol{X}_{-, k+1}+\boldsymbol{K}_{k+1}\left(\boldsymbol{z}_{k+1}-\boldsymbol{z}_{-, k+1}\right) \\
\boldsymbol{P}_{+, k+1}= & \boldsymbol{P}_{-, k+1}-\boldsymbol{K}_{k+1} \boldsymbol{P}_{z z, k+1} \boldsymbol{K}_{k+1}^{T} .
\end{aligned}
$$


where $d, d_{1}, d_{2}=1, \cdots, m, i=1, \cdots, n . \boldsymbol{P}_{z z, k+1}^{d_{1} d_{2}}$ indicates the components of the covariance matrix of the measurements and $\boldsymbol{P}_{X z, k+1}^{i d}$ denotes the components of the cross-covariance matrix between the state and the measurements.

Note that nonlinear JT-HEKF filters are based on the aforementioned filtering process and implemented in the JT scheme. The main properties of JT-HEKF- $\kappa$ algorithms include: 1) to retain the nonlinear information of the dynamical and measurement models during the filtering process; 2) to save computational time.

Let us assume that the mean and covariance of the state and parameter vectors are known at time $t_{k}$, this is: the $n$-dimensional state vector $\boldsymbol{x}_{+, k}$, the $\boldsymbol{l}$-dimensional parameter vector $\boldsymbol{p}_{+, k}$, and the complete covariance matrix $\boldsymbol{P}_{+, k}$ are provided. In particular, $\boldsymbol{P}_{+, k}$ includes the state covariance submatrix $\boldsymbol{P}_{+, k}^{x}$, the parameter covariance sub-matrix $\boldsymbol{P}_{+, k}^{p}$, and the covariance sub-matrix $\boldsymbol{P}_{+, k}^{x p}$ associated to the state and parameter vectors,

$$
\boldsymbol{P}_{+, k}=\left(\begin{array}{cc}
\boldsymbol{P}_{+, k}^{\boldsymbol{x}} & \boldsymbol{P}_{+, k}^{\boldsymbol{x p}} \\
\boldsymbol{P}_{+, k}^{\boldsymbol{p}} & \boldsymbol{P}_{+, k}^{\boldsymbol{p}}
\end{array}\right), \quad \text { with } \quad \boldsymbol{P}_{+, k}^{\boldsymbol{p x}}=\left(\boldsymbol{P}_{+, k}^{\boldsymbol{x p}}\right)^{T}
$$

Expanding (20) around the mean of the augmented state up to order $\kappa$, in terms of (8), we obtain the prediction of the augmented state at time $t_{k+1}$ by computing the expectation of the polynomial gives

$$
\begin{aligned}
\boldsymbol{X}_{-, k+1}^{i} & =E\left\{\overline{\boldsymbol{X}}_{k+1}^{i}+\mathcal{P}_{\overline{\boldsymbol{X}}_{k+1}^{i}}^{\kappa}\left(\delta \boldsymbol{X}_{k}\right)\right\} \\
& =\sum_{0 \leq \gamma_{1}+\cdots+\gamma_{n+l} \leq \kappa} \boldsymbol{a}_{\gamma_{1} \ldots \gamma_{n+l}}^{i} E\left\{\delta X_{k, 1}^{\gamma_{1}} \cdots \delta X_{k, N+l}^{\gamma_{n+l}}\right\},
\end{aligned}
$$

where the superscript $i \in[1, n+l]$ gives the index of the augmented state vector, $\delta \boldsymbol{X}_{k}=\left[\delta \boldsymbol{x}_{k}^{T}, \delta \boldsymbol{p}_{k}^{T}\right]^{T}$ indicates the deviations on the spacecraft state and parameters respectively, $\overline{\boldsymbol{X}}_{k+1}^{i}=\boldsymbol{\Phi}_{k+1}^{i}\left(t_{k+1} ; t_{k}, \boldsymbol{X}_{+, k}\right)$ indicates the nominal state prediction, and

$$
\boldsymbol{a}_{\gamma_{1} \ldots \gamma_{n+l}}^{i}=\frac{1}{\gamma !} \frac{\partial^{\gamma} \boldsymbol{\Phi}_{k+1}^{i}}{\partial \delta X_{k, 1}^{\gamma_{1}} \cdots \partial \delta X_{k, n+l}^{\gamma_{n+l}}} .
$$

Although the estimated paramters are merged into the augmented state, it is worth to mention two special cases for the parameter prediction. In the 
first case it is assumed that the estimated parameters are fixed, this is, (19) is invariable and degraded into

$$
\boldsymbol{p}_{-, k+1}^{j}=\boldsymbol{p}_{+, k}^{j} .
$$

In essence, one does not need to propagate the parameters together with the spacecraft state in this case, which can save computational time since it reduces the dimension of the estimation problem. This case will be further discussed when the position of the tracking station is estimated.

In the second case, we consider that the parameter equations (19) are nonlinear. A formulation similar to (8) is used to predict the parameter vector

$$
\begin{aligned}
\boldsymbol{p}_{-, k+1}^{j} & =E\left\{\overline{\boldsymbol{p}}_{k+1}^{j}+\mathcal{P}_{\overline{\boldsymbol{p}}_{k+1}^{j}}^{\kappa}\left(\delta \boldsymbol{p}_{k}\right)\right\} \\
& =\sum_{0 \leq \gamma_{n+1}+\cdots+\gamma_{n+l} \leq \kappa} \boldsymbol{b}_{\gamma_{n+1} \ldots \gamma_{n+l}}^{j} E\left\{\delta p_{k, 1}^{\gamma_{n+1}} \ldots \delta p_{k, l}^{\gamma_{n+l}}\right\}
\end{aligned}
$$

where the coefficients of the Taylor expansions are

$$
\boldsymbol{b}_{\gamma_{n+1} \ldots \gamma_{n+l}}^{j}=\frac{1}{\gamma !} \frac{\partial^{\gamma} \boldsymbol{\Phi}_{p, k+1}^{j}}{\partial \delta p_{k, 1}^{\gamma_{n+1}} \cdots \partial \delta p_{k, l}^{\gamma_{n+l}}} .
$$

Analogously, the prediction of the $d$-th measurement can be obtained through the computation of the expectations of the polynomial results,

$$
\begin{aligned}
\boldsymbol{z}_{-, k+1}^{d} & =E\left\{\overline{\boldsymbol{z}}_{k+1}^{d}+\mathcal{P}_{\overline{\boldsymbol{z}}_{k+1}^{d}}^{\kappa}\left(\delta \boldsymbol{X}_{k}\right)\right\} \\
& =\sum_{0 \leq \gamma_{1}+\cdots+\gamma_{n+l} \leq \kappa} \boldsymbol{c}_{\gamma_{1} \ldots \gamma_{n+l}}^{d} E\left\{\delta X_{k, 1}^{\gamma_{1}} \cdots \delta X_{k, n+l}^{\gamma_{n+l}}\right\}
\end{aligned}
$$

where $d \in[1, m]$ indicates the index of the measurement equations, $\overline{\boldsymbol{z}}_{k+1}^{d}=$ $\boldsymbol{\Xi}_{k+1}^{d}\left(t_{k+1} ; t_{k}, \boldsymbol{X}_{-, k+1}\right)$, and

$$
\boldsymbol{c}_{\gamma_{1} \ldots \gamma_{n+l}}^{d}=\frac{1}{\gamma !} \frac{\partial^{\gamma} \boldsymbol{\Xi}_{k+1}^{d}}{\partial \delta X_{k, 1}^{\gamma_{1}} \cdots \partial \delta X_{k, n+l}^{\gamma_{n+l}}} .
$$

Inserting Taylor polynomials (24), (26) and (27) into the filtering process (22) and (23), one can obtain the detailed JT-HKEF- $\kappa$ filtering algorithms:

- Prediction equations: at time $t_{k+1}$,

$$
\boldsymbol{X}_{-, k+1}^{i}=\sum_{0 \leq \gamma_{1}+\cdots+\gamma_{n+l} \leq \kappa} \boldsymbol{a}_{\gamma_{1} \ldots \gamma_{n+l}}^{i} E\left\{\delta X_{k, 1}^{\gamma_{1}} \cdots \delta X_{k, N+l}^{\gamma_{n+l}}\right\}
$$




$$
\begin{aligned}
& \boldsymbol{z}_{-, k+1}^{d}=\sum_{0 \leq \gamma_{1}+\cdots+\gamma_{n+l} \leq \kappa} \boldsymbol{c}_{\gamma_{1} \ldots \gamma_{n+l}}^{d} E\left\{\delta X_{k, 1}^{\gamma_{1}} \cdots \delta X_{k, n+l}^{\gamma_{n+l}}\right\}, \\
& \boldsymbol{P}_{-, k+1}^{x, i_{1} i_{2}}=\sum_{1 \leq \gamma_{1}+\cdots+\gamma_{n+l} \leq \kappa 1 \leq \tilde{\gamma}_{1}+\cdots+\tilde{\gamma}_{n+l} \leq \kappa} \boldsymbol{a}_{\gamma_{1} \ldots \gamma_{n+l}}^{i_{1}} \boldsymbol{a}_{\tilde{\gamma}_{1} \ldots \tilde{\gamma}_{n+l}}^{i_{2}} . \\
& E\left\{\delta x_{k, 1}^{\gamma_{1}+\tilde{\gamma}_{1}} \cdots \delta x_{k, n}^{\gamma_{n}+\tilde{\gamma}_{n}} \delta p_{k, 1}^{\gamma_{n+1}+\tilde{\gamma}_{n+1}} \cdots \delta p_{k, l}^{\gamma_{n+l}+\tilde{\gamma}_{n+l}}\right\} \\
& -\delta \boldsymbol{m}_{k+1}^{x, i_{1}} \delta \boldsymbol{m}_{k+1}^{x, i_{2}}+E\left\{\boldsymbol{w}_{k}^{i_{1}} \boldsymbol{w}_{k}^{i_{2}}\right\}, \quad i_{1}, i_{2} \in[1, n], \\
& \boldsymbol{P}_{-, k+1}^{x p, i j}=\sum_{1 \leq \gamma_{1}+\cdots+\gamma_{n+l} \leq \kappa} \sum_{1 \leq \tilde{\gamma}_{n+1}+\cdots+\tilde{\gamma}_{n+l} \leq \kappa} \boldsymbol{a}_{\gamma_{1} \ldots \gamma_{n+l}}^{i} \boldsymbol{b}_{\tilde{\gamma}_{n+1} \ldots \tilde{\gamma}_{n+l}}^{j} . \\
& E\left\{\delta x_{k, 1}^{\gamma_{1}} \cdots \delta x_{k, n}^{\gamma_{n}} \delta p_{k, 1}^{\gamma_{n+1}+\tilde{\gamma}_{n+1}} \cdots \delta p_{k, l}^{\gamma_{n+l}+\tilde{\gamma}_{n+l}}\right\} \\
& -\delta \boldsymbol{m}_{k+1}^{x, i} \delta \boldsymbol{m}_{k+1}^{p, j}, \quad i \in[1, n], j \in[1, l] \text {, } \\
& \boldsymbol{P}_{-, k+1}^{p, j_{1} j_{2}}=\sum_{1 \leq \gamma_{n+1}+\cdots+\gamma_{n+l} \leq \kappa} \sum_{1 \leq \tilde{\gamma}_{n+1}+\cdots+\tilde{\gamma}_{n+l} \leq \kappa} \boldsymbol{b}_{\gamma_{n+1} \ldots \gamma_{n+l}}^{j_{1}} \boldsymbol{b}_{\tilde{\gamma}_{n+1} \ldots \tilde{\gamma}_{n+l}}^{j_{2}} . \\
& E\left\{\delta p_{k, 1}^{\gamma_{n+1}+\tilde{\gamma}_{n+1}} \cdots \delta p_{k, l}^{\gamma_{n+l}+\tilde{\gamma}_{n+l}}\right\}-\delta \boldsymbol{m}_{k+1}^{p, j_{1}} \delta \boldsymbol{m}_{k+1}^{p, j_{2}} \\
& +E\left\{\boldsymbol{v}_{k}^{j_{1}} \boldsymbol{v}_{k}^{j_{2}}\right\}, \quad j_{1}, j_{2} \in[1, l],
\end{aligned}
$$

where

$$
\delta \boldsymbol{m}_{k+1}^{x, i}=\overline{\boldsymbol{x}}_{k+1}^{i}-\boldsymbol{x}_{-, k+1}^{i}, \quad \delta \boldsymbol{m}_{k+1}^{p, i}=\overline{\boldsymbol{p}}_{k+1}^{i}-\boldsymbol{p}_{-, k+1}^{i},
$$

and the covariance matrix of the augmented state is

$$
\boldsymbol{P}_{-, k+1}=\left(\begin{array}{ll}
\boldsymbol{P}_{-, k+1}^{x} & \boldsymbol{P}_{-, k+1}^{x p} \\
\boldsymbol{P}_{-, k+1}^{p x} & \boldsymbol{P}_{-, k+1}^{p}
\end{array}\right) .
$$

- Update equations: incorporting the new measurement $\boldsymbol{z}_{k+1}$ at time $t_{k+1}$ into the estimation algorithm,

$$
\begin{aligned}
& \boldsymbol{P}_{z z, k+1}^{d_{1} d_{2}}=\sum_{1 \leq \gamma_{1}+\cdots+\gamma_{n+l} \leq \kappa} \sum_{1 \leq \tilde{\gamma}_{1}+\cdots+\tilde{\gamma}_{n+l} \leq \kappa} \boldsymbol{c}_{\gamma_{1} \ldots \gamma_{n+l}}^{d_{1}} \boldsymbol{c}_{\gamma_{1} \ldots \gamma_{n+l}}^{d_{2}} . \\
& E\left\{\delta x_{k, 1}^{\gamma_{1}+\tilde{\gamma}_{1}} \cdots \delta x_{k, n}^{\gamma_{n}+\tilde{\gamma}_{n}} \delta p_{k, 1}^{\gamma_{n+1}+\tilde{\gamma}_{n+1}} \cdots \delta p_{k, l}^{\gamma_{n+l}+\tilde{\gamma}_{n+l}}\right\} \\
& -\delta \boldsymbol{n}_{k+1}^{d_{1}} \delta \boldsymbol{n}_{k+1}^{d_{2}}+E\left\{\boldsymbol{u}_{k+1}^{d_{1}} \boldsymbol{u}_{k+1}^{d_{2}}\right\}, \quad d_{1}, d_{2} \in[1, m], \\
& \boldsymbol{P}_{x z, k+1}^{i d}=\sum_{1 \leq \gamma_{1}+\cdots+\gamma_{n+l} \leq \kappa} \sum_{1 \leq \tilde{\gamma}_{1}+\cdots+\tilde{\gamma}_{n+l} \leq \kappa} \boldsymbol{a}_{\gamma_{1} \ldots \gamma_{n+l}}^{i} \boldsymbol{c}_{\tilde{\gamma}_{1} \ldots \tilde{\gamma}_{n+l}}^{d} . \\
& E\left\{\delta x_{k, 1}^{\gamma_{1}+\tilde{\gamma}_{1}} \cdots \delta x_{k, n}^{\gamma_{n}+\tilde{\gamma}_{n}} \delta p_{k, 1}^{\gamma_{n+1}+\tilde{\gamma}_{n+1}} \cdots \delta p_{k, l}^{\gamma_{n+l}+\tilde{\gamma}_{n+l}}\right\} \\
& -\delta \boldsymbol{m}_{k+1}^{x, i} \delta \boldsymbol{n}_{k+1}^{d}, \quad i \in[1, n], d \in[1, m],
\end{aligned}
$$




$$
\begin{aligned}
\boldsymbol{P}_{p z, k+1}^{j d}= & \sum_{1 \leq \gamma_{n+1}+\cdots+\gamma_{n+l} \leq \kappa} \sum_{1 \leq \tilde{\gamma}_{1}+\cdots+\tilde{\gamma}_{n+l} \leq \kappa} \boldsymbol{b}_{\gamma_{n+1} \ldots \gamma_{n+l}}^{j} \boldsymbol{c}_{\tilde{\gamma}_{1} \ldots \tilde{\gamma}_{n+l}}^{d} . \\
& E\left\{\delta x_{k, 1}^{\tilde{\gamma}_{1}} \cdots \delta x_{k, n}^{\tilde{\gamma}_{n}} \delta p_{k, 1}^{\gamma_{n+1}+\tilde{\gamma}_{n+1}} \cdots \delta p_{k, l}^{\gamma_{n+l}+\tilde{\gamma}_{n+l}}\right\} \\
& -\delta \boldsymbol{m}_{k+1}^{p, j} \delta \boldsymbol{n}_{k+1}^{d}, \quad j \in[1, l], d \in[1, m],
\end{aligned}
$$

where $\delta \boldsymbol{n}_{k+1}^{d}=\overline{\boldsymbol{z}}_{k+1}^{d}-\boldsymbol{z}_{-, k+1}^{d}$. Using (29) and (30), we can obtain an augmented matrix $\boldsymbol{P}_{X z, k+1}=\left[\boldsymbol{P}_{x z, k+1}^{T} \boldsymbol{P}_{p z, k+1}^{T}\right]^{T}$.

Finally, we can update the estimated state and parameter vectors, as well as the covariance matrix, incorporating the measurement information at time $t_{k+1}$. So, at time $t_{k+1}$

$$
\begin{aligned}
& \boldsymbol{K}_{k+1}=\boldsymbol{P}_{X z, k+1}\left(\boldsymbol{P}_{z z, k+1}\right)^{-1} \\
& \boldsymbol{X}_{+, k+1}=\boldsymbol{X}_{-, k+1}+\boldsymbol{K}_{k+1}\left(\boldsymbol{z}_{k+1}-\boldsymbol{z}_{-, k+1}\right), \\
& \boldsymbol{P}_{+, k+1}=\boldsymbol{P}_{-, k+1}-\boldsymbol{K}_{k+1} \boldsymbol{P}_{z z, k+1} \boldsymbol{K}_{k+1}^{T} .
\end{aligned}
$$

It is clear that, due to the nonlinearity of the system, at time $t_{k+1}$ the distributions of the state and parameter vectors in the JT-HEKF- $\kappa$ filters (excluding JT-HEKF-1) are no longer Gaussian, even that their distributions at time $t_{k}$ were Gaussian. Although reference [7] showed that the Gaussian assumption is accurate enough in the orbit determination application using the proposed JT-HEKF- $\kappa$ filters, in a forthcomming work we will remove this assumption. Under the Gaussian assumption, Isserlis's formula [18] provides an analytical way to compute the associated expectation values in the JTHEKF- $\kappa$ algorithms.

As it has already been said, the purpose of this paper is to show how some parameters can be accurately estimated jointly with the spacecraft state. The nature of the parameters can be very different, from physical ones related with the spacecraft to the uncertain ones associated with the measurement procedure. Without loss of generality, and in order to show the behavior of the developed methodology, here we only present two illustrative cases: one physically affected by the time-varying area-to-mass ratio, and another one related with an uncertain tracking station position.

\subsubsection{Case study A: spacecraft state and physical parameter estimation}

Although many spacecraft physical parameters are pre-designed and precisely manufactured for concrete missions, still some of them may be determined or adjusted during the mission, and need to be estimated in real time. Examples of such ones could be the spacecraft mass and the illuminated 
cross sectional area. The uncertainty on the mass is mainly originated from unaccurate operations of the propulsion system, while the uncertainty on the illuminated cross sectional area is affected by the shape and attitude of the spacecraft, as well as the relative position between the spacecraft and the Sun.

In general, the modeling error of the SRP acceleration is the largest dynamical error source in the GEO regime [10]. Morever, due to maneuvers, the area-to-mass ratio $\eta=A / m$ changes with time and needs refitting. In this case study we assume that we are inside a period of time without maneuvers, but we have a time varying $\eta$ because the non-spherical GEO spacecraft is rotating with respect to the Sun in a diurnal basis, and so, its cross sectional area varies accordingly. The nonlinear variation law considered in the simulation is assumed to be

$$
\dot{\eta}=\eta_{0} \sin \left(\omega_{e} t\right)
$$

where $\omega_{e}$ stands for the average angular speed of the Earth's rotation.

\subsubsection{Case study B: spacecraft state and tracking station position estimation}

For usual data collection techniques, underlying systematic biases and unmodeled observation errors significantly degrade the measurement quality. These facts limit the orbit determination accuracy, especially for the GEO case, where one arcsecond of angular error from the ground tracking station position, corresponds to approximately 200 meters of the spacecraft position observational error.

Therefore, the precise estimation of these systematic biases and unmodeled observation errors, as well as the offset to remove them, is of significant interest and importance. In particular, the accurate estimation of the height of a removable ground tracking station is meaningful, since it is difficult to be accurately determined via the GPS services. In this case study we attempt to precisely estimate the uncertain position of a ground tracking station. In general, the position of the ground tracking station is provided by specifying its east longitude $\Lambda$, geodetic latitude $\phi$, and an elevation $H$ above the WGS84 ellipsoidal surface, and the position vector has to be converted into the ECI frame. Unlike the previous case, (19) is now fixed and simplifies into (25). Therefore, assuming that the height of the ground tracking station is denoted by $H$ and its corresponding uncertainty by $\delta H$, the parameter equation can be expressed as

$$
H_{k+1}=H_{k}+\delta H_{k}+v_{k} .
$$




\subsubsection{Sensitivity analysis}

Complementing the previous discussions for the case studies A and B, we focus on the sensitivity analysis of the JT-HEKF- $\kappa$ filters with respect to initial estimation errors, measurement acquisition frequencies, and the observation geometry between the spacecraft and the ground tracking station (i.e., $\Delta \Lambda_{r}=\lambda-\Lambda$ ). In general, the classical EKF filtering algorithms lose accuracy in the low measurement acquisition frequency case. Therefore, three different measurement acquisition frequencies are considered in order to produce some significant conclusions. On the other hand, we analyze the sensitivity of JT-HEKF- $\kappa$ algorithms with respect to the initial simulation conditions subjected to a large estimation error distribution (i.e., large values of $\sigma_{r}, \sigma_{v}$, and $\sigma_{p}$ ) via Monte Carlo (MC) simulations. It is worth mentioning that we omit the simulation with small initial estimation error distribution case, since both the usual EKF and the JT-HEKF proposed in this paper work very well for the simultaneous estimation of the spacecraft state and additional parameters in the GEO regime. Finally, a sensitivity analysis with respect to the observational geometry (25 uniformly distributed sampling points are taken from the interval $\left.\Delta \Lambda_{r} \in\left[-60^{\circ},-60^{\circ}\right]\right)$ is also implemented via $\mathrm{MC}$ simulations.

In order to assess the sensitivity tests of the JT-HEKF- $\kappa$ filtering algorithms, some statistical indices are defined as follows,

$$
\begin{gathered}
\kappa_{\kappa} \bar{\varepsilon}_{i}^{N_{p}}=\frac{\sum_{j=1}^{N_{p}} \varepsilon_{i}^{j}}{N_{p}}, \quad \tau_{\kappa_{1}}^{\kappa_{2}}=\frac{\kappa_{2} \bar{\varepsilon}_{i}^{N_{p}}}{\kappa_{\kappa_{1}} \bar{\varepsilon}_{i}^{N_{p}}}, \\
{ }_{\kappa}^{\bar{\varepsilon}} \sigma_{i}^{N_{p}}=\sqrt{\frac{\sum_{j=1}^{N_{p}}\left(\varepsilon_{i}^{j}-{ }_{\kappa} \bar{\varepsilon}_{i}^{N_{p}}\right)^{2}}{N_{p}-1}}, \quad \zeta_{\kappa_{1}}^{\kappa_{2}}=\frac{{ }_{\bar{\kappa}} \sigma_{2}^{N_{p}}}{{ }_{\kappa_{1}} \sigma_{i}^{N_{p}}} .
\end{gathered}
$$

where $\varepsilon_{i}^{j}, i=r, v, p$ indicates the position error, velocity error and parameter error of the $j$ th Monte Carlo simulation calculated at the steady stage of the filtering process, ${ }_{\kappa} \bar{\varepsilon}_{i}^{N_{p}}$ and ${ }_{\kappa}^{\bar{\varepsilon}} \sigma_{i}^{N_{p}}$ denote the mean and the standard deviation of $\varepsilon_{i}^{j}$ accounting for the expansion order $\kappa, N_{p}$ is the total number of Monte Carlo simulations. Note that $\tau_{\kappa_{1}}^{\kappa_{2}}$ reveal the error ratio between JT-HEKF- $\kappa_{2}$ and JT-HEKF- $\kappa_{1}$ filtering algorithms. if $0<\tau_{\kappa_{1}}^{\kappa_{2}}<1$, JT-HEKF- $\kappa_{2}$ algorithm features the better accuracy than JT-HEKF- $\kappa_{1}$ algorithm, otherwise is worse. The ratio $\zeta_{\kappa_{1}}^{\kappa_{2}}$ shows the dispersion of the estimation error around 
the mean. If $\zeta_{\kappa_{1}}^{\kappa_{2}}>1$, JT-HEKF- $\kappa_{2}$ algorithm possesses a higher dispersion than JT-HEKF- $\kappa_{1}$ algorithm, otherwise is smaller.

\section{Results}

Currently, optical telescopes are typically exploited to track the GEO objects. As a passive data collection technique, optical telescopes enable to efficiently track numerous objects surreptitiously. The recent development of more powerful wide-field-of-view optical sensors possesses sub-arcsecond observation accuracy for the near-geosynchronous objects [12]. In particular, the state of the art accuracy of the most sophisticated optical sensors enables to reach 20 milli-arcseconds [19]. In the following simulations, we assume that the measurement errors on the topocentric right ascension (RA, $\alpha$ ) and declination $(\delta)$ are Gaussian white noises with the same standard deviation 66.6 milli-arcseconds (corresponding to $\sigma_{m}=3.232 \times 10^{-7} \mathrm{rad}$ and $14 \mathrm{~m}$ in the GEO regime). The position of the ground tracking station is known by specifying its east longitude $\Lambda$, geodetic latitude $\phi$, and an elevation $H$ above the WGS84 ellipsoidal surface. The local sidereal time of the ground tracking station is $s_{o}=\Lambda+G A(t)$. After the coordinate transformation, the position of the ground tracking station is expressed in the ECI frame as $\boldsymbol{r}_{\boldsymbol{o}}=\left(x_{o}, y_{o}, z_{o}\right)=\left(R_{c} \cos \phi \cos s_{o}, R_{c} \cos \phi \sin s_{o}, R_{s} \sin \phi\right)$, where $\boldsymbol{r}_{\boldsymbol{o}}$ indicates the inertial position vector of the tracking station, while $R_{c}$ and $R_{s}$ are two intermediate values depending on the equatorial radius, $R_{e}$, flattening, $f$, and height of the tracking station, $H$. The spacecraft position vector relative to the ground tracking station is expressed as $\varrho=\boldsymbol{r}-\boldsymbol{r}_{o}=\left(x-x_{o}, y-y_{o}, z-z_{o}\right)$. Therefore, the measurement vector $\boldsymbol{z}=(\alpha, \delta)$ can be computed by the corresponding measurement equations,

$$
\left\{\begin{array}{l}
\alpha=\arctan \left(\frac{y-y_{o}}{x-x_{o}}\right)+u_{1}, \\
\delta=\arcsin \left(\frac{z-z_{o}}{\|\varrho\|_{2}}\right)+u_{2} .
\end{array}\right.
$$

In practice, these measurements are provided by optical telescopes or radars; here we numerically generate such inputs according to the following procedure: we assume a true nominal trajectory of the higher fidelity dynamical model (including $10 \times 10$ EGM96S gravity model, SRP, albedo, Sun and Moon gravitational perturbations) that is propagated forward to time $t_{k}$, 
Table 1: True initial values including spacecraft state, parameters, and the ground tracking station position

\begin{tabular}{cccc}
\hline Parameter & Nominal value & Initial state & Nominal value \\
\hline Epoch & 15 November $2015,0: 0: 0$ UTC & $x_{0}$ & $24487.8 \mathrm{~km}$ \\
$\eta=A / m$ & $74 / 3300 \mathrm{~m}^{2} / \mathrm{kg}$ & $y_{0}$ & $34324.4 \mathrm{~km}$ \\
$C_{r}$ & 1.3 & $z_{0}$ & $0 \mathrm{~km}$ \\
$\Lambda$ & $42.0516528^{\circ}$ & $\dot{x}_{0}$ & $-2.50298 \mathrm{~km} / \mathrm{s}$ \\
$\phi$ & $0.7293472^{\circ}$ & $\dot{y}_{0}$ & $1.78568 \mathrm{~km} / \mathrm{s}$ \\
$H$ & $1620 \mathrm{~m}$ & $\dot{z}_{0}$ & $0 \mathrm{~km} / \mathrm{s}$ \\
\hline
\end{tabular}

where the observational measurement is done; then, numerical measurement at this epoch is generated by adding some Guassian white noises into the spacecraft topocentric right ascension RA $(\alpha)$ and declination $(\delta)$, which is calculated in terms of the true spacecraft state from the above state propagation and the true position of the tracking station (32). Table 1 shows the true initial spacecraft state vector and parameters, as well as the true tracking station position considered in the simulations.

In order to compare the performances of the Cartesian and GEO representations, the JT-HEKF- $\kappa$ filtering algorithms are implemented in both models with the same simulation conditions. The performance of a JTHEKF- $\kappa$ filter includes the estimation accuracy, computational burden and its robustness. In particular, the estimation error is defined as the difference between the estimated state and parameter vectors (i.e., $\boldsymbol{x}_{+, k}, \boldsymbol{p}_{+, k}$ ), computed by the JT-HEKF- $\kappa$ filtering algorithms, and the true state and parameter vectors (i.e., $\boldsymbol{x}_{k}, \boldsymbol{p}_{k}$ ), obtained by the numerical integration of the state and parameter equations, this is, $\boldsymbol{\varepsilon}_{\boldsymbol{x}}=\boldsymbol{x}_{+, k}-\boldsymbol{x}_{k}$, and $\boldsymbol{\varepsilon}_{\boldsymbol{p}}=\boldsymbol{p}_{+, k}-\boldsymbol{p}_{k}$. Furthermore, we define the position error $\varepsilon_{r}=\sqrt{\varepsilon_{x}^{2}+\varepsilon_{y}^{2}+\varepsilon_{z}^{2}}$ and velocity error $\varepsilon_{\boldsymbol{v}}=\sqrt{\varepsilon_{\dot{x}}^{2}+\varepsilon_{\dot{y}}^{2}+\varepsilon_{\dot{z}}^{2}}$. For reasons of space and clarity, only the sensitivity analysis results for case study A are given, the same conclusions are also valid for case study B. All the codes are written in $\mathrm{C}++$ compiled with gcc version 5.5.0 and implemented with a laptop processor Intel(R) Core(TM) i5-7300HQ under Linux.

\subsection{Spacecraft physical parameter estimation}

In this study case we consider all the spacecraft physical parameter equations, the state and measurement equations are nonlinear. The performances 
of the JT-HEKF- $\kappa$ filtering algorithms, implemented in both coordinate representations, are assessed by the simultaneous estimation of the state $\boldsymbol{x}$ and the time-varying area-to-mass ratio $\eta$.

We assume that the initial guess of the area-to-mass ratio is $0.1 \mathrm{~m}^{2} / \mathrm{kg}$ off from the true value given in Table 1 , and its initial standard deviation is $\sigma_{\eta}=0.1 \mathrm{~m}^{2} / \mathrm{kg}$. The initial estimation errors are of $100 \mathrm{~km}$ in the position vector components, and of $0.5 \mathrm{~m} / \mathrm{s}^{2}$ in the velocity vector components. The adopted initial covariance matrix is

$$
\boldsymbol{P}_{+, 0}=\left(\begin{array}{cc}
\boldsymbol{P}_{+, 0}^{x} & \mathbf{0} \\
\mathbf{0} & \sigma_{\eta}^{2}
\end{array}\right), \quad \text { where } \quad \boldsymbol{P}_{+, 0}^{x}=\left(\begin{array}{cc}
10^{10} \boldsymbol{I}_{3 \times 3} & \mathbf{0} \\
\mathbf{0} & 0.25 \boldsymbol{I}_{3 \times 3}
\end{array}\right) .
$$

The total simulation time is 4 days, provided that 7 measurements can be done per night (10 hours per day), separated by regular time intervals. Let the angle measurement noise be a zero mean white noise with standard deviation $\sigma_{m}=3.232 \times 10^{-7} \mathrm{rad}$ (corresponding to $14 \mathrm{~m}$ in the GEO regime). The measurement noise matrix $\boldsymbol{R}$ is

$$
\boldsymbol{R}=\left(\begin{array}{cc}
\sigma_{m}^{2} & 0 \\
0 & \sigma_{m}^{2}
\end{array}\right)
$$

Figures 1 and 2 exhibit the results of the simultaneous estimation of the area-to-mass ratio and the spacecraft state, respectively implemented in the Cartesian and GEO representations. Both figures show that the JT-HEKF-2 filter outputs a better accuracy than the JT-HEKF-1 filter (i.e., the classic EKF filter). For both JT-HEKF-2 and JT-HEKF-3 filters, the position and velocity estimation errors are respectively less than $10 \mathrm{~m}$ and $5 \times 10^{-4} \mathrm{~m} / \mathrm{s}$. The estimation error of the area-to-mass ratio converges to $2 \times 10^{-4} \mathrm{~m}^{2} / \mathrm{kg}$, which is around $0.1 \%$ of the nominal value. In contrast, the JT-HEKF-1 filter produces the worse estimation errors of the spacecraft position, velocity, and area-to-mass ratio: respectively, $100 \mathrm{~m}, 3 \times 10^{-3} \mathrm{~m} / \mathrm{s}$ and $3 \times 10^{-3} \mathrm{~m}^{2} / \mathrm{kg}$ (14\%). In essence, the JT-HEKF-1 algorithm fails in the estimation of the area-to-mass ratio, as is shown in the bottom line sub-figures of Figs. 1 and 2. Both sub-figures illustrate that the area-to-mass ratio estimation error is almost $14 \%$ of the initial error, so the filter does not work properly. From this simulation, it follows that we can at least obtain one order of magnitude accuracy gain replacing the linear EKF (i.e., JT-HEKF-1 filter) by the JTHEKF-2 filter. Furthermore, due to normality hypothesis, the expectations of the third order terms vanish, thus no accuracy gain is obtained replacing the JT-HEKF-2 algorithm by the JT-HEKF-3 one. 

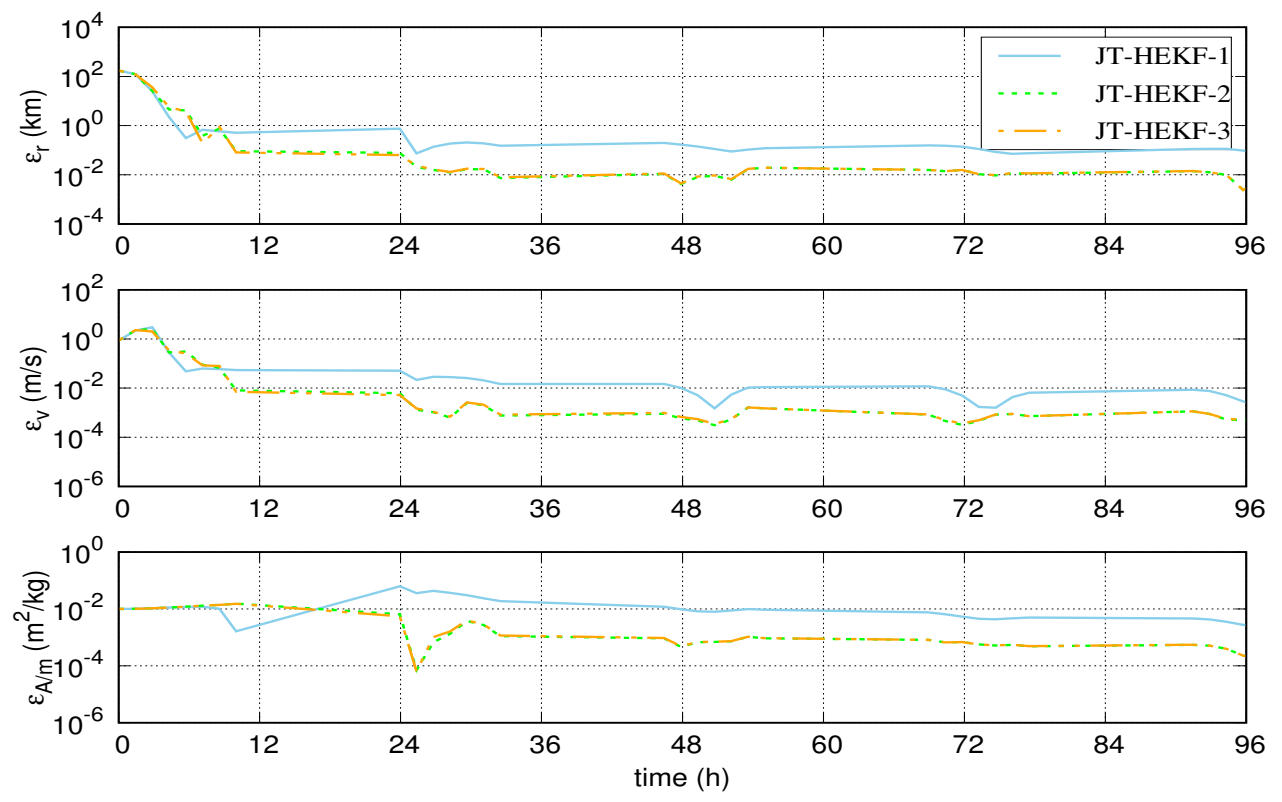

Figure 1: Case A: State and area-to-mass ratio estimation errors with a measurement frequency 7 times/night. Implemented in the GEO representation.
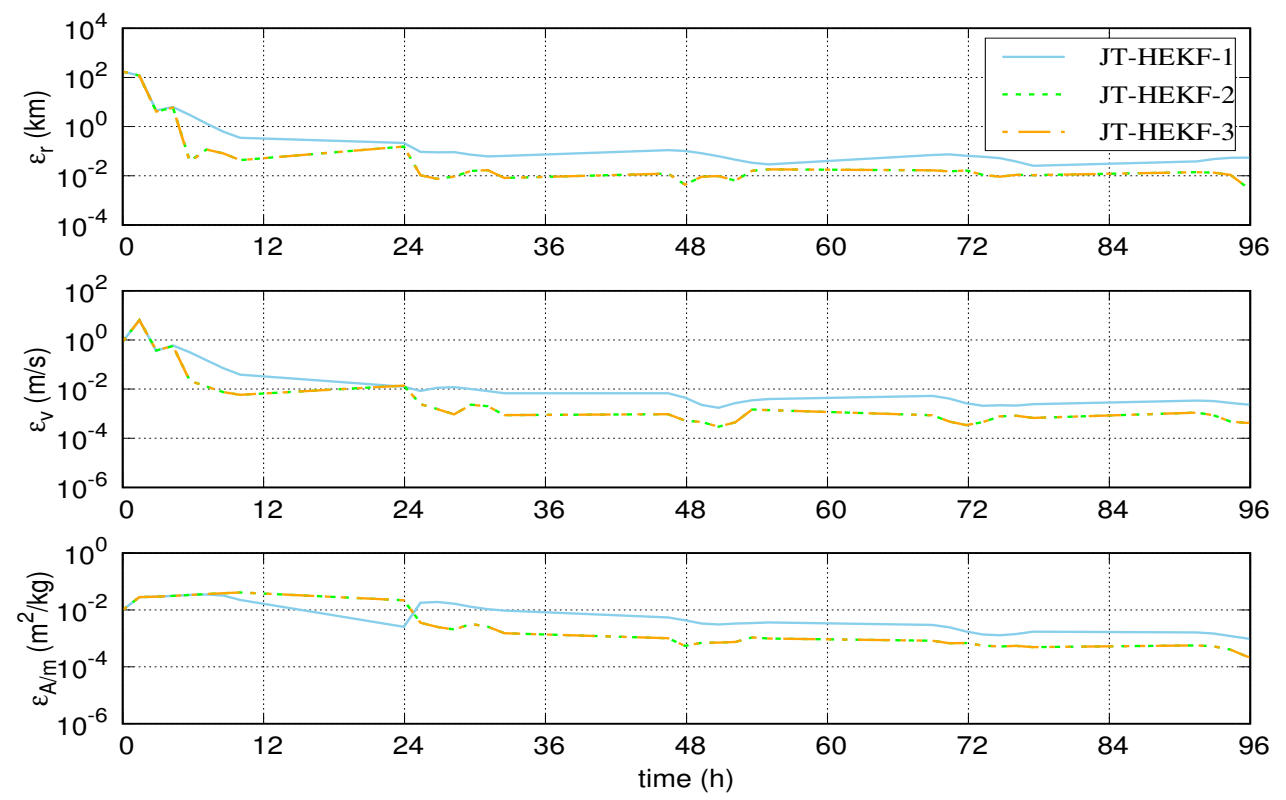

Figure 2: Case A: State and area-to-mass ratio estimation errors with a measurement frequency 7 times/night. Implemented in the Cartesian representation. 
Comparing Fig. 1 with Fig. 2, it follows that the accuracies of both representations are of the same order of magnitude but, in general, the accuracy of GEO representation is a little better than that of the Cartesian representation if the algorithms are convergent (i.e., JT-HEKF-2 and JT-HEKF-3). This phenomenon originates from the more accurate prediction of the GEO representation in the time-update step. Although the Cartesian representation behaves better than GEO representation in the JT-HEKF-1 algorithm, it makes no sense since the 1st order algorithm is essentially failing.

Another important issue to be pointed out here is related with the computational efficiency. Table 2 shows the computational cost of the simulations corresponding to Figs. 1 and 2 (without including the computational cost for generating the measurements). It underlines that the GEO representation is much faster than the Cartesian one, since the GEO elements vary much slower than the classical Cartesian ones in the propagation of GEO orbits. Then, a larger integration step size is adopted in the prediction process, resulting in less computational time and in a higher efficiency. Therefore, one can conclude that GEO representation possesses a better performance (including a superior estimation accuracy and less computational burden) than the Cartesian representation. This conclusion is also validated in the case study B. But, for brevity, in what follows we only show the results of the GEO representation for the case study B, while the ones corresponding to the Cartesian representation are omitted.

Table 2: Computational time (s)

\begin{tabular}{cccc}
\hline \multirow{2}{*}{ Model } & \multicolumn{3}{c}{ JT-HEKF- $\kappa$} \\
\cline { 2 - 4 } & $\kappa=1$ & $\kappa=2$ & $\kappa=3$ \\
\hline GEO & 24 & 44 & 114 \\
Cartesian & 144 & 149 & 197 \\
\hline
\end{tabular}

\subsubsection{Sensitivity analysis with respect to the observational geometry}

Clearly, in order to estimate the spacecraft state and area-to-mass ratio, the GEO spacecraft must be in the visible region from the ground tracking station. The sensitivity analysis of the JT-HEKF- $\kappa$ algorithms with respect to the observational geometry is implemented, in particular with respect to the longitude difference $\Delta \Lambda_{r}$ between the spacecraft and the tracking station. For this purpose, we take 25 uniform sampling points in the interval 

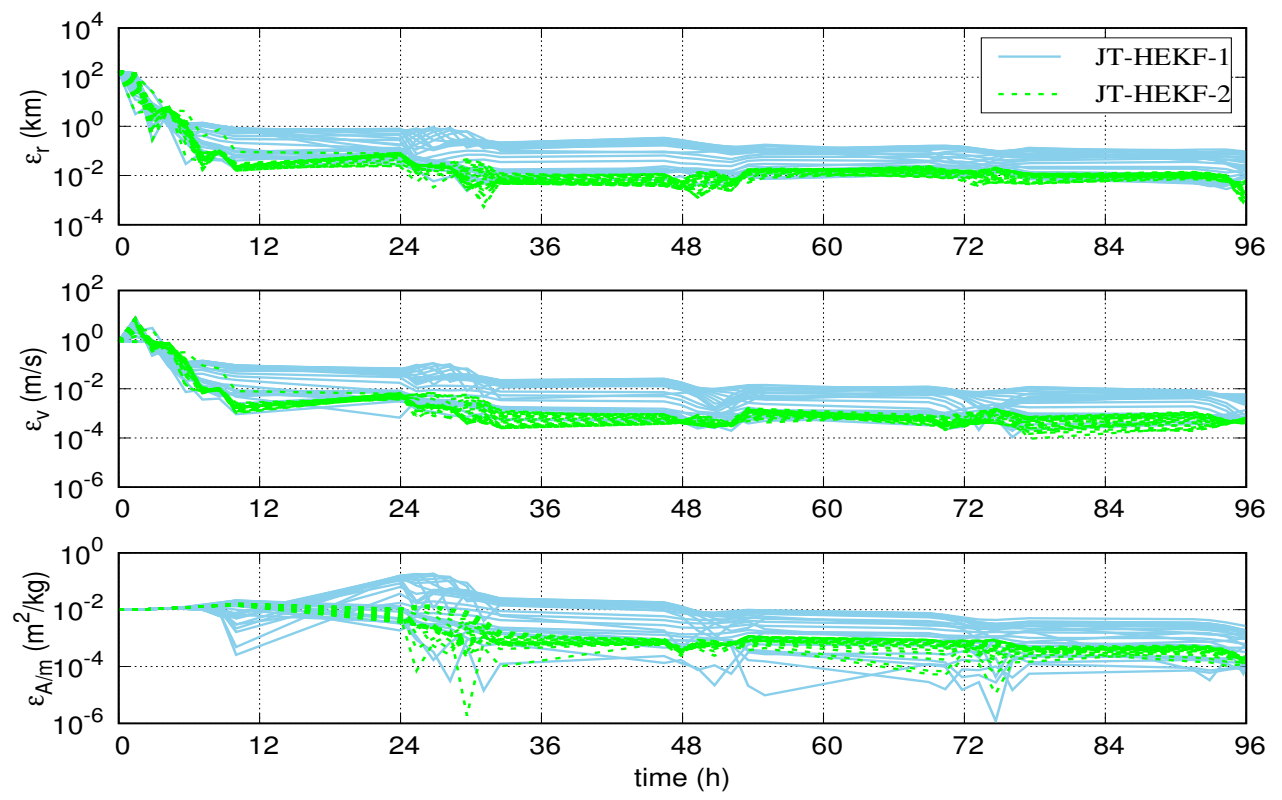

Figure 3: Case A: Sensitivity analysis with respect to the observational geometry with a measurement frequency of 7 times/night. Implemented in the GEO representation.

Table 3: Case A: Sensitivity analysis to the observational geometry

\begin{tabular}{ccccc}
\hline & Indices & $i=r$ & $i=v$ & $i=\eta$ \\
& Unit & $\mathrm{m}$ & $10^{-4} \mathrm{~m} / \mathrm{s}$ & $10^{-4} \mathrm{~m}^{2} / \mathrm{kg}$ \\
\hline \multirow{2}{*}{ JT-HEKF-1 } & ${ }_{1} \bar{\varepsilon}_{i}^{25}$ & 36.7 & 27.6 & 7.96 \\
& ${ }_{1} \sigma_{i}^{25}$ & 24.1 & 421.9 & 438.5 \\
JT-HEKF-2 & ${ }_{2} \bar{\varepsilon}_{i}^{25}$ & 7.88 & 6.45 & 1.50 \\
& ${ }_{2}{ }_{2}^{25}$ & 2.08 & 76.8 & 81.7 \\
\hline \multirow{2}{*}{ Ratio } & $\tau_{1}^{2}={ }_{2} \bar{\varepsilon}_{i}^{25} /{ }_{1} \bar{\varepsilon}_{i}^{25}$ & 0.215 & 0.234 & 0.188 \\
& $\zeta_{1}^{2}={ }_{2}^{\bar{\varepsilon}} \sigma_{i}^{25} /{ }_{1}^{\bar{\varepsilon}} \sigma_{i}^{25}$ & 0.086 & 0.182 & 0.186 \\
\hline
\end{tabular}


$\left.\Delta \Lambda_{r} \in\left[-60^{\circ},-60^{\circ}\right]\right)$ to implement a $\mathrm{MC}$ simulation. Apart from the different initial longitude of the spacecraft, the same initial conditions and the same measurements are used.

Figure 3 summarizes the results and provides a deep insight into the sensitivity of the JT-HEKF- $\kappa$ algorithms with respect to the observational geometry. The results of JT-HEKF-3 filter are omitted, since no accuracy improvement is obtained relative to the JT-HEKF-2 filter. It is clear that the mean accuracy of the JT-HEKF-2 filter is better than the one of JT-HEKF-1 filter, also the standard deviation of the estimation errors at the steady stage of the JT-HEKF-2 filter is much smaller than the one of the JT-HEKF-1 filter, as shown in Table 3. Therefore, we can conclude the accuracy of the JT-HEKF-1 filter is more strongly dependent on the observational geometry than the one of the JT-HEKF-2 filter, being less robust.

\subsubsection{Sensitivity analysis with respect to the initial estimation errors}

To analyze the sensitivity with respect to initial estimation errors, we carry out a Monte Carlo simulation with 2000 sampling points generated around the initial nominal state subjected to multivariable Guassian distribution. The furthest 25 sampling points, where the nonlinearities play a prominent role, are taken as initial state estimates. Both Fig. 4 and Table 4 exhibit the mean and the standard deviation of the estimation errors, computed by means of JT-HEKF-1 and JT-HEKF-2 filtering algorithms. It is apparent that the JT-HEKF-2 filter outputs a better accuracy and a smaller error dispersion, displaying a much more robust performance with respect to initial estimation errors.

Using radar measurements (including the range information and the line of sight directions of the spacecraft), we can introduce the range information into the measurement models (32) and explore if it improves the performances of the JT-HEKF- $\kappa$ procedures. The simulation results indicate that the addition of range measurement leads to a more severe divergence for the original divergent filter (this is, for JT-HEKF-1), while it produces an accu-

racy improvement for the original convergent ones (this is, for JT-HEKF-2 and JT-HEKF-3), as shown in the Appendix.

\subsubsection{Sensitivity analysis with respect to the measurement acquisition fre- quency}

To illustrate this sensitivity we consider three different measurement acquisition frequencies: 7, 14 and 21 times per night. In principle, high mea- 

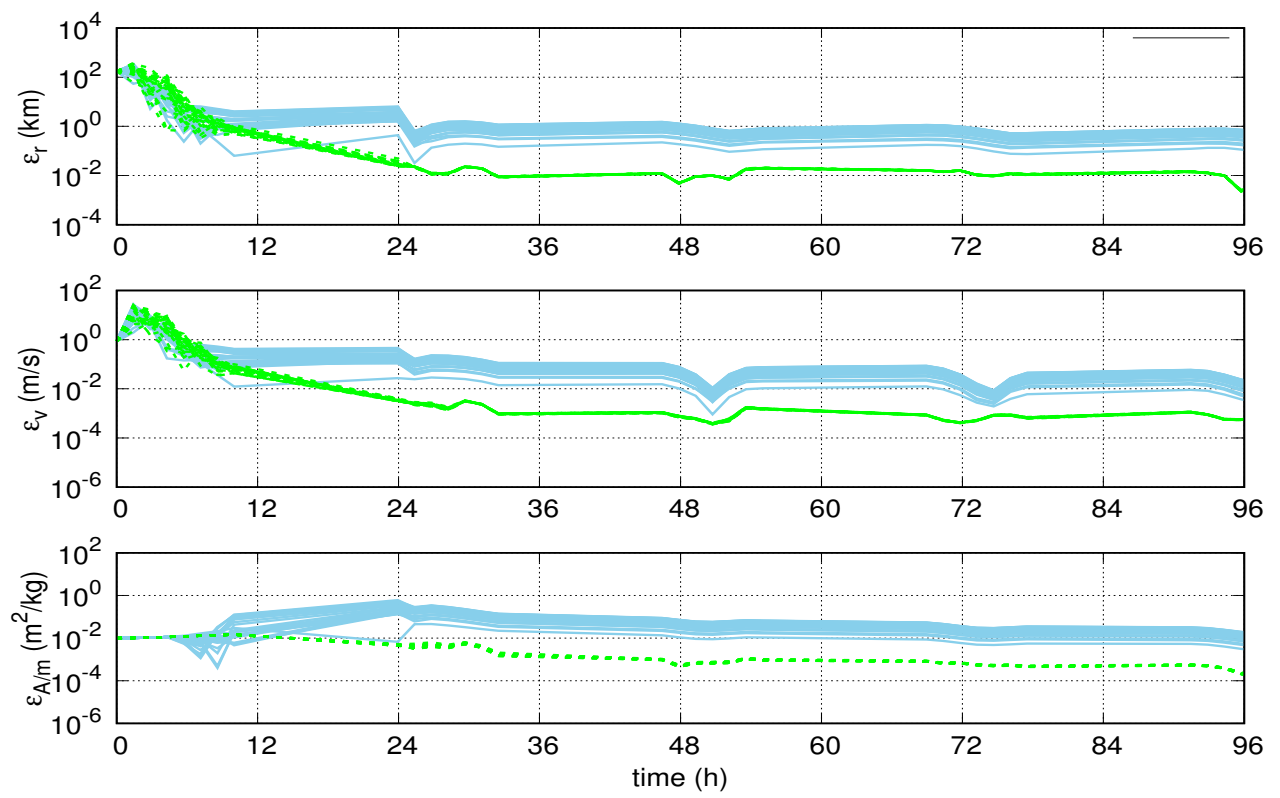

Figure 4: Case A: Sensitivity analysis with respect to initial estimation errors with a measurement frequency of 7 times/night. Implemented in the GEO representation.

Table 4: Case A: Sensitivity analysis with respect to initial estimation errors

\begin{tabular}{ccccc} 
& Indices & $i=r$ & $i=v$ & $i=\eta$ \\
& Unit & $\mathrm{m}$ & $10^{-4} \mathrm{~m} / \mathrm{s}$ & $10^{-4} \mathrm{~m}^{2} / \mathrm{kg}$ \\
\hline \multirow{2}{*}{ JT-HEKF-1 } & ${ }_{1} \bar{\varepsilon}_{i}^{25}$ & 296.9 & 79.0 & 101.2 \\
& ${ }_{1}^{\bar{\varepsilon}} \sigma_{i}^{25}$ & 117.7 & 317.6 & 315.5 \\
JT-HEKF-2 & ${ }_{2} \bar{\varepsilon}_{i}^{25}$ & 7.76 & 7.25 & 1.82 \\
& ${ }_{2} \sigma_{i}^{25}$ & 0.176 & 71.8 & 77.4 \\
\hline \multirow{2}{*}{ Ratio } & $\tau_{1}^{2}={ }_{2} \bar{\varepsilon}_{i}^{25} /{ }_{1} \bar{\varepsilon}_{i}^{25}$ & 0.026 & 0.092 & 0.018 \\
& $\zeta_{1}^{2}={ }_{2}{ }_{2} \sigma_{i}^{25} /{ }_{1}{ }^{\bar{\varepsilon}} \sigma_{i}^{25}$ & 0.001 & 0.226 & 0.245 \\
\hline
\end{tabular}


surement acquisition frequency is in favor of the accuracy improvement of the JT-HEKF- $\kappa$ filters, especially for the low order filter JT-HEKF-1 since the linearization of the state equations always tend to lose accuracy for long integration time spans(i.e., in the low frequency acquisition case). Generally speaking, high order nonlinear approximations of the state equations are capable of maintaining a highly precise uncertainty propagation describing the localized motion around the nominal trajectory.
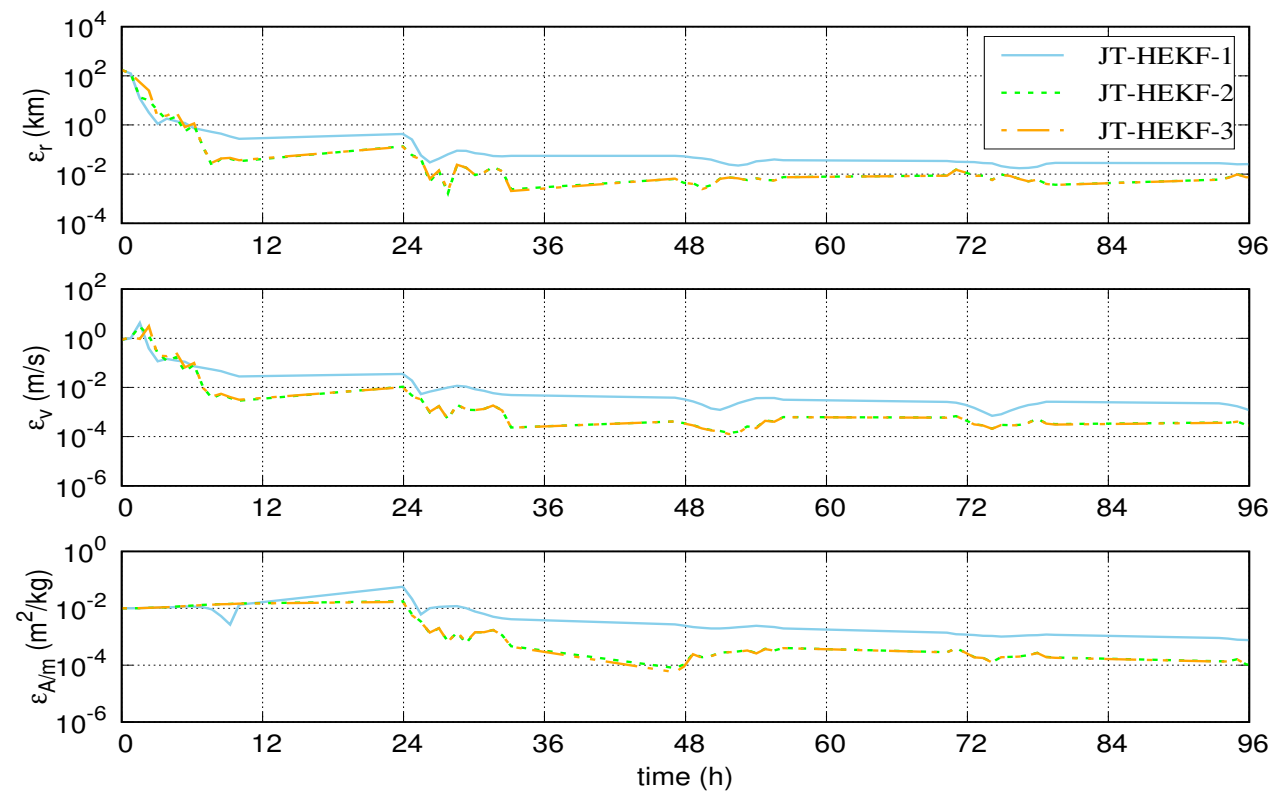

Figure 5: Case A: State and area-to-mass ratio estimation errors with a measurement frequency 14 times/night. Implemented in GEO representation.

Figures 5 and 6 respectively display the simulation results with measurement frequencies of 14 and 21 times per night, separated by regular time intervals. The comparison among Figs. 1, 5, and 6 illustrates that a high measurement acquisition frequency benefits for the accuracy improvement in all JT-HEKF algorithms at all orders. We also note that the low order JT-HEKF filter can obtain a larger accuracy gain than the high order ones. This is, high order JT-HEKF algorithms have a stronger robustness when the tracking sensors encounter functional degradation (i.e., when the measurement acquisition frequency decreases significantly). Finally, we mention that the corresponding sensitivity analyses implemented in the Cartesian representation produce the similar results, but omitted here for brevity. 

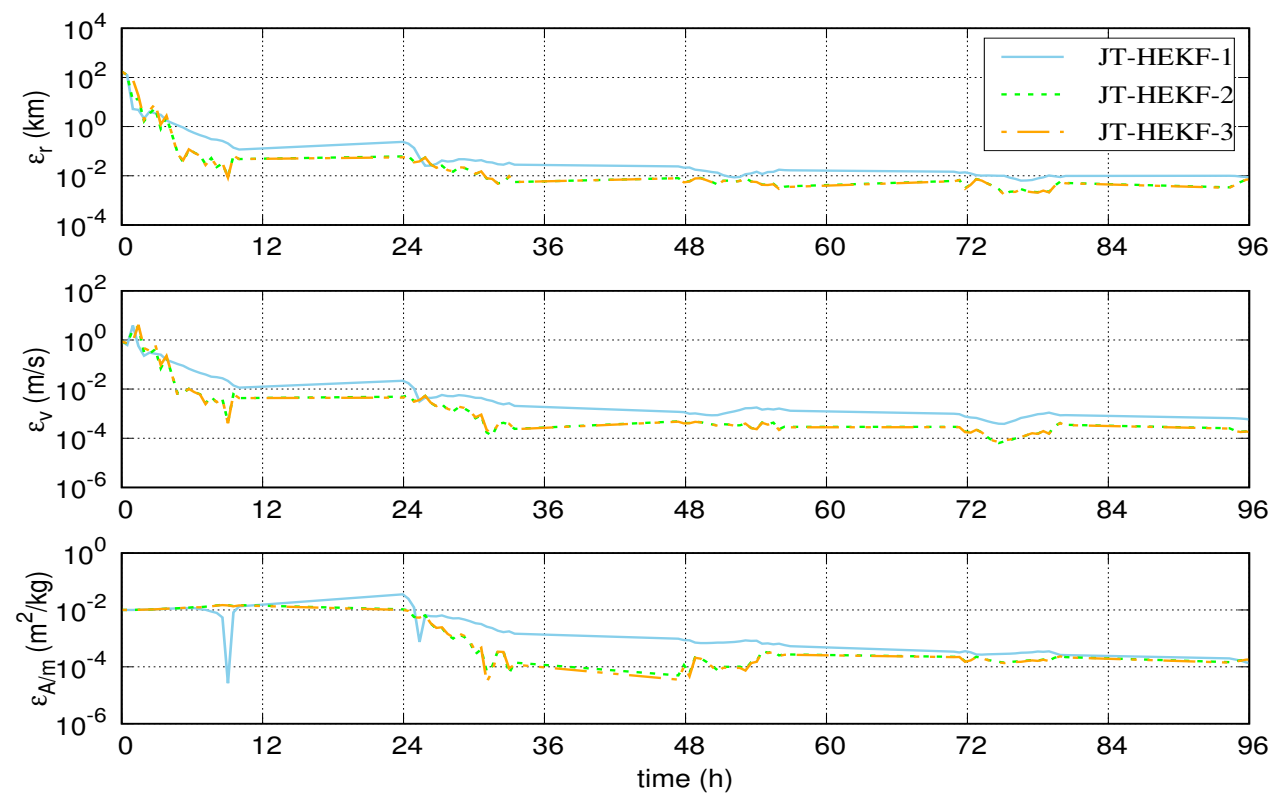

Figure 6: Case A: State and area-to-mass ratio estimation errors with a measurement frequency 21 times/night. Implemented in GEO representation.
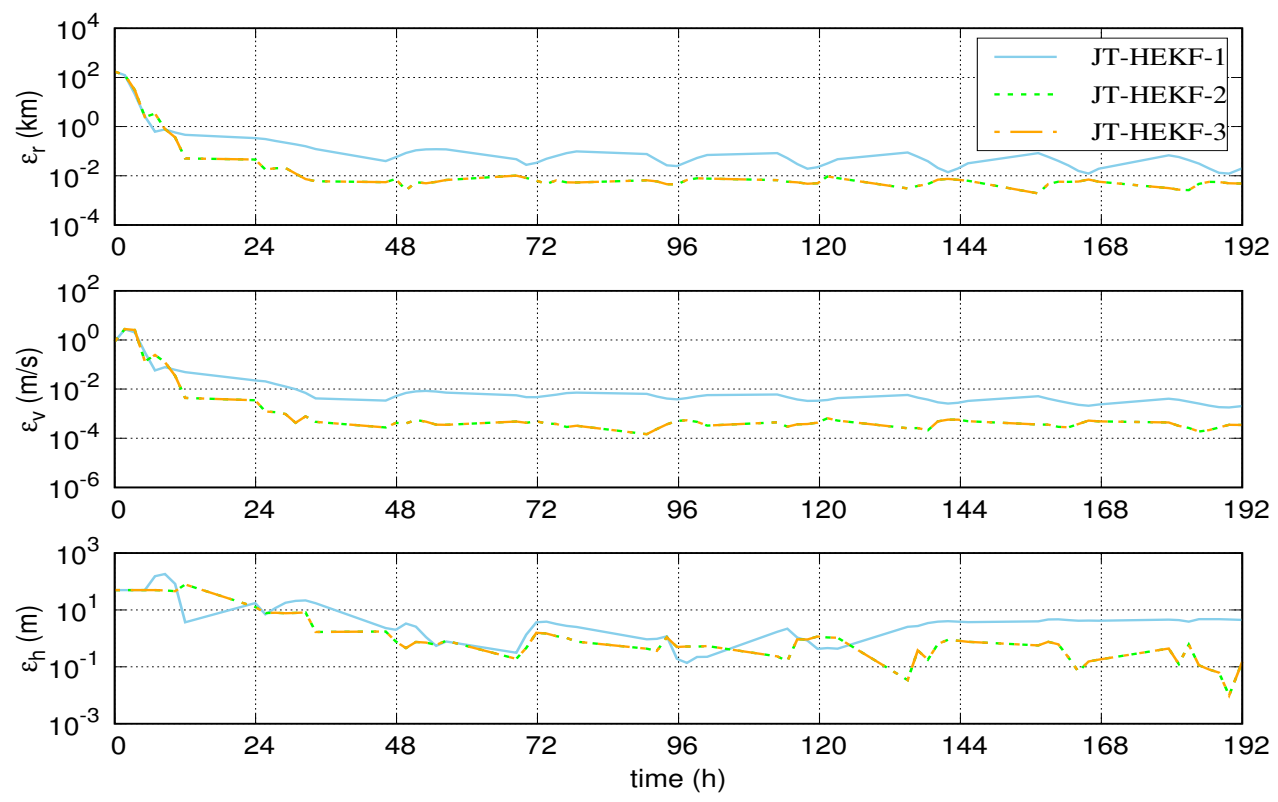

Figure 7: Case B: State and height estimation errors with a measurement frequency 7 times/night. Implemented in the GEO representation. 


\subsection{Tracking station position estimation}

The preceding discussions indicate that the JT-HEKF- $\kappa$ algorithms are capable of estimating the spacecraft state and parameters simultaneously. In this subsection we discuss the feasibility to estimate an uncertain position of a ground tracking station. Clearly, the underlying systematic biases and unmodeled observational errors always lead to a significant degeneration of the measurement quality. In this simulation we manage to estimate systematic biases of a ground tracking station and, subsequently, remove them in order to improve the accuracy of the measurement equations. In general, accurate GPS services provide very accurate values of the longitude $\Lambda$ and the geodetic latitude $\phi$, but not so accurate for the height $H$. Therefore, this issue is specially meaningful in cases where the tracking stations are not fixed. In the following simulation, we try to determine both the spacecraft state $\boldsymbol{x}$ and an uncertain height $H$ of the tracking station. The true initial state and parameters of the spacecraft, as well as the precise position of the tracking station, are given in Table 1.

Assume that the initial estimation error of the ground tracking station height is $50 \mathrm{~m}$ off from the true height assumed in Table 1 , this is, $\delta H_{0}=$ $50 \mathrm{~m}$, and that the standard deviation of the initial height error is $\sigma_{H}=50 \mathrm{~m}$. The initial state estimation errors are of $100 \mathrm{~km}$ in all the components of the position vector, and of $0.5 \mathrm{~m} / \mathrm{s}$ in velocity vector components. We consider no errors on the longitude and latitude of the tracking station. Thus, the adopted initial covariance matrix is,

$$
\boldsymbol{P}_{+, 0}=\left(\begin{array}{cc}
\boldsymbol{P}_{+, 0}^{x} & \mathbf{0} \\
\mathbf{0} & \sigma_{H}^{2}
\end{array}\right), \quad \text { where } \quad \boldsymbol{P}_{+, 0}^{x}=\left(\begin{array}{cc}
10^{10} \boldsymbol{I}_{3 \times 3} & \mathbf{0} \\
\mathbf{0} & 0.25 \boldsymbol{I}_{3 \times 3}
\end{array}\right) .
$$

The measurement acquisition frequency is taken again as 7 times per night separated by regular time intervals. The measurement equations (32) are still adopted and the corresponding measurement noise covariance matrix is (33). The accuracy comparison of the first, second and third orders JTHEKF filters, for estimating the spacecraft state and the uncertain height of the ground tracking station, are shown in Fig. 7. It shows that the nonlinear JT-HEKF- $\kappa$ algorithms $(\kappa>1)$ work very well in the estimation process of both state and parameter. For the JT-HEKF-2 and JT-HEKF-3 filters, the position and velocity estimation errors are respectively around $5 \mathrm{~m}$ and $3 \times 10^{-4} \mathrm{~m} / \mathrm{s}$. The estimation error of the height converges to $2 \mathrm{~m}$, which is around $4 \%$ of the initial estimation error. Again, the JT-HEKF-1 filter 
produces worse estimation errors for position, velocity, and height, respectively: $70 \mathrm{~m}, 0.003 \mathrm{~m} / \mathrm{s}$ and $5 \mathrm{~m}(10 \%)$. In principle, the JT-HEKF-2 and JT-HEKF-3 algorithms are capable of extracting more nonlinear information from the dynamical system improving the estimation accuracy, at least one order of magnitude, relative to the JT-HEKF-1 filter. In addition, the results implemented in the Cartesian representation produce the same conclusions as for the GEO representation, but omitted for brevity.

\section{Conclusions}

This paper studies jet transport-based high order extended Kalman filtering algorithms, implemented both in Cartesian and hybrid geosynchronous orbit element representations, for the simultaneous estimation of spacecraft state and additional parameters in the geosynchronous regime. These parameters describe either the spacecraft physical features or the position information of the ground tracking station. The effectiveness of the proposed nonlinear filtering estimators has been validated considering two case examples.

The comparisons between the two implementations underline that the implementation of hybrid geosynchronous orbit elements not only possesses better estimation accuracy, but also needs of less computational cost than the Cartesian one. Letting aside the performance comparisons between the two coordinate representations, the simulations also confirm that both coordinate implementations are effective to achieve real-time simultaneous spacecraft state and parameter estimation via the proposed nonlinear Kalman filtering algorithms.

The performance comparisons of the nonlinear filtering estimators at different orders are also investigated. The results show that higher order filters provide a superior accuracy estimation, and usually, one order of magnitude accuracy gain can be easily obtained in the two case studies considered, just by tuning the filters from 1st to 2nd order. Furthermore, a series of detailed sensitivity analyses of the proposed nonlinear filters with respect to the observational geometry, the initial estimation errors, and the measurement acquisition frequency are carried out. One can conclude that the nonlinear jet transport-based high order filters (i.e., $\kappa>1$ ) output a smaller estimation error dispersion relative to the classical extended Kalman filter, pointing to that the proposed nonlinear filters are more robust than the usual extended Kalman filter. Finally, it should be mentioned that the proposed jet 
transport-based high order filtering algorithms could be suitable to carry out future applications related with high precise geostationary or geosynchronous orbit determination.

\section{Appendix}

To incorporate more measurement information, the range from the spacecraft to the tracking station is assumed to be available. The standard deviation of the measurement error for the range is supposed to be 1 meter. A Monte Carlo simulation is implemented to assess its performance. To compare the results with Figure 4, the JT-HEKF- $\kappa$ algorithms are fed with the same measurements (excluding the range information), and initialized with the same initial estimates of the state and area-to-mass ratio. Figure 8 shows that the supplement of the range measurement information decreases the performance of JT-HEKF-1 filter, while improves the performances of the JT-HEKF-2 and JT-HEKF-3 filters. Tables 4 and 5 provide more detailed information. Their comparison further reveals that more measurement information makes the original divergent algorithm, this is JT-HEKF-1, diverge now more severely, but it improves the estimation accuracy of the original convergent methods (i.e. the JT-HEKF-2 and JT-HEKF-3 filters).

Table 5: Case A: Sensitivity analysis with respect to initial estimation errors incorporating the range measurement information

\begin{tabular}{|c|c|c|c|c|}
\hline & Indices & $i=r$ & $i=v$ & $i=\eta$ \\
\hline & Unit & $\mathrm{m}$ & $10^{-3} \mathrm{~m} / \mathrm{s}$ & $10^{-4} \mathrm{~m}^{2} / \mathrm{kg}$ \\
\hline \multirow{2}{*}{ JT-HEKF-1 } & ${ }_{1} \bar{\varepsilon}_{i}^{25}$ & 204.5 & 288.0 & 83.2 \\
\hline & ${ }_{1}^{\bar{\varepsilon}} \sigma_{i}^{25}$ & 77.5 & 1953.0 & 2147.0 \\
\hline \multirow{2}{*}{ JT-HEKF-2 } & ${ }_{2} \bar{\varepsilon}_{i}^{25}$ & 1.22 & 0.80 & 0.18 \\
\hline & ${ }_{2}^{\bar{\varepsilon}} \sigma_{i}^{25}$ & 0.07 & 11.64 & 12.27 \\
\hline \multirow{2}{*}{ Ratio } & $\tau_{1}^{2}={ }_{2} \bar{\varepsilon}_{i}^{25} /{ }_{1} \bar{\varepsilon}_{i}^{25}$ & 0.006 & 0.003 & 0.002 \\
\hline & $\zeta_{1}^{2}={ }_{2}^{\bar{\varepsilon}} \sigma_{i}^{25} /{ }_{1}^{\bar{\varepsilon}} \sigma_{i}^{25}$ & 0.001 & 0.006 & 0.006 \\
\hline
\end{tabular}



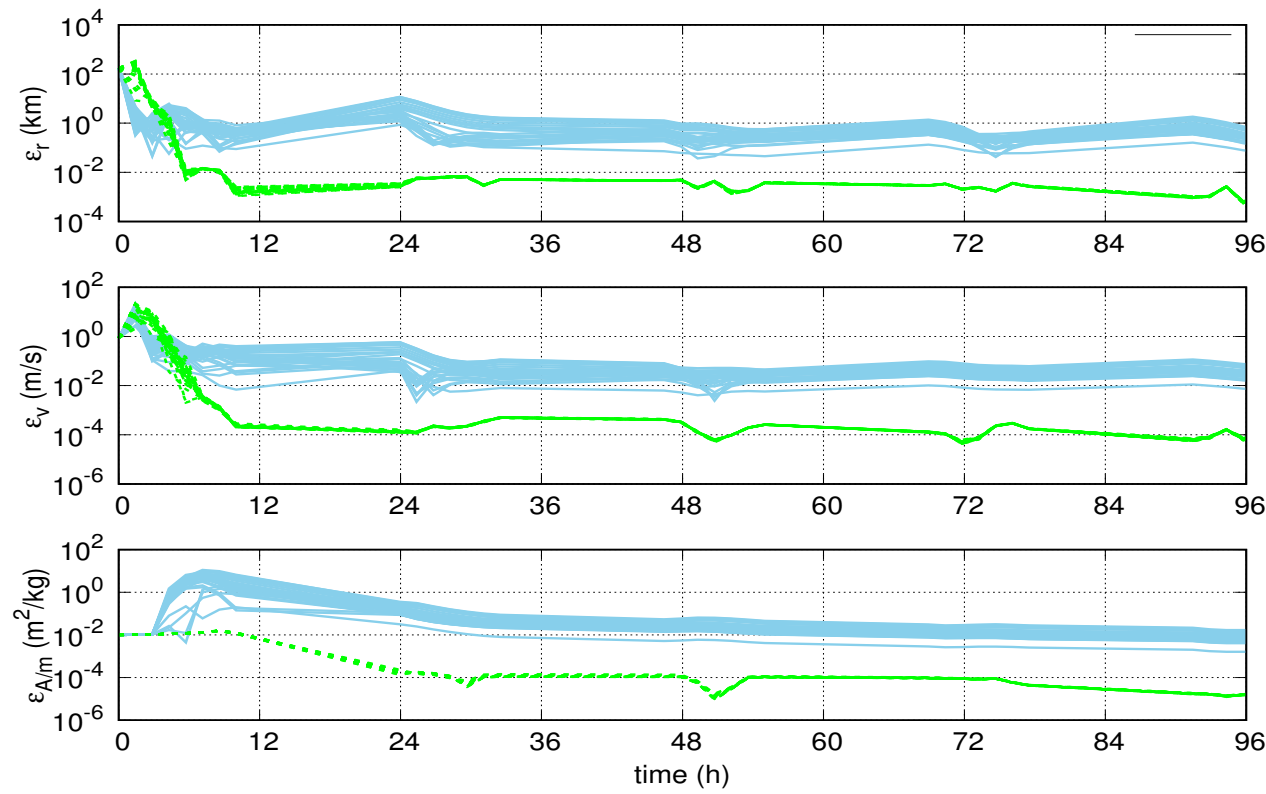

Figure 8: Case A: Sensitivity analysis with respect to initial estimation errors with a measurement frequency of 7 times/night. Implemented in the GEO representation and incorporating the range measurement information.

\section{Acknowledgments}

J.C. thanks the support of Doctorate Foundation of Northwestern Polytechnical University and the Chinese Scholarship Council. He also thanks National natural science foundation of China for the grant 11572248, MINECOFEDER for the grant MTM2015-65715-P and Key Laboratory of Aerospace Flight Dynamics for the grant 2015afdl015. J.J.M. thanks MINECO-FEDER for the grant MTM2015-65715-P and the Catalan government for the grant 2017SGR-1049. G.G. thanks the Catalan grant government 2017SGR-1374, and MINECO-FEDER for the grant MTM2016-80117-P.

\section{References}

[1] R. M. Fuster, M. F. Usón, A. B. Ibars, Interferometric orbit determination for geostationary satellites, Science China Information Sciences 60 (6) (2017) 060302 (2017). doi:10.1007/s11432-016-9052-y.

[2] S. Ribo, J. C. Arco, E. Cardellach, S. Oliveras, A. Rius, C. Buck, Preliminary results of digital satellite tv opportunity signals scattered on 
the sea-surface, in: Workshop on Reflectometry using GNSS and Other Signals of Opportunity (GNSS+R), Purdue University, West Lafayette, USA, October 10-11, 2012 (October 10-11, 2012).

[3] M. Valli, R. Armellin, P. di Lizia, M. R. Lavagna, Nonlinear filtering methods for spacecraft navigation based on differential algebra, Acta Astronautica 94 (1) (2014) 363 - 374 (2014). doi:10.1016/j.actaastro.2013.03.009.

[4] R. Armellin, P. Di Lizia, R. Zanetti, Dealing with uncertainties in anglesonly initial orbit determination, Celestial Mechanics and Dynamical Astronomy 125 (4) (2016) 435-450 (2016). doi:10.1007/s10569-016-9694-z.

[5] F. Cavenago, P. Di Lizia, M. Massari, A. Wittig, On-board spacecraft relative pose estimation with high-order extended kalman filter, Acta Astronautica 158 (2019) 55-67 (2019).

[6] E. A. Wan, R. Van Der Merwe, The unscented kalman filter for nonlinear estimation, in: Adaptive Systems for Signal Processing, Communications, and Control Symposium 2000. AS-SPCC. The IEEE 2000, Ieee, 2000, pp. 153-158 (2000). doi:10.1109/ASSPCC.2000.882463.

[7] R. S. Park, D. J. Scheeres, Nonlinear semi-analytic methods for trajectory estimation, Journal of guidance, control, and dynamics 30 (6) (2007) 1668-1676 (2007). doi:10.2514/1.29106.

[8] J. L. Crassidis, J. L. Junkins, Optimal estimation of dynamic systems, CRC press, Boca Raton, FL, 2004, Ch. 5, pp. 243-320 (2004).

[9] O. Montenbruck, E. Gill, Satellite orbits: models, methods and applications, Springer Science \& Business Media, 2012, Ch. 3, pp. 53-81 (2012).

[10] C. Tang, X. Hu, S. Zhou, R. Guo, et al., Improvement of orbit determination accuracy for beidou navigation satellite system with two-way satellite time frequency transfer, Advances in Space Research 58 (7) (2016) 1390-1400 (2016). doi:10.1016/j.asr.2016.06.007.

[11] E. M. Soop, Handbook of geostationary orbits, Kluwer Academic, Dordrecht, The Netherlands, 1994, Ch. 2, pp. 13-26 (1994). 
[12] J. Tombasco, Orbit estimation of geosynchronous objects via groundbased and space-based optical tracking, Ph.D. thesis, University of Colorado at Boulder (2011).

[13] J. Tombasco, P. Axelrad, M. Jah, Specialized coordinate representation for dynamic modeling and orbit estimation of geosynchronous orbits, Journal of guidance, control, and dynamics 33 (6) (2010) 1824-1836 (2010). doi:10.2514/1.48903.

[14] J. Chen, J. J. Masdemont, G. Gómez, J. Yuan, High accuracy state and parameter estimation of geo-stationary satellites using jet transport based nonlinear filtering algorithm, in: 69th International Astronautical Congress, Bremen, Germany, 2018 (2018).

[15] M. Berz, Modern map methods in particle beam physics, Vol. 108, Academic Press, 1999, Ch. 2, pp. 81-111 (1999).

[16] D. Pérez-Palau, Dynamical transport mechanisms in celestial mechanics and astrodynamics problems, Ph.D. thesis, Universitat de Barcelona (2016).

[17] D. Pérez-Palau, J. J. Masdemont, G. Gómez, Tools to detect structures in dynamical systems using jet transport, Celestial Mechanics and Dynamical Astronomy 123 (3) (2015) 1-24 (2015).

[18] L. Isserlis, On a formula for the product-moment coefficient of any order of a normal frequency distribution in any number of variables, Biometrika 12 (1/2) (1918) 134-139 (1918). doi:10.2307/2331932.

[19] A. T. Tokunaga, New generation ground-based optical/infrared telescopes, in: Encyclopedia of the Solar System (Third Edition), Elsevier, 2014, pp. 1089-1105 (2014). doi:10.1016/B978-0-12-415845-0.00051-7. 